TRANSACTIONS OF THE

AMERICAN MATHEMATICAL SOCIETY

Volume 364, Number 4, April 2012, Pages 2157-2191

S 0002-9947(2011)05546-7

Article electronically published on December 1, 2011

\title{
FINITE EULER PRODUCTS AND THE RIEMANN HYPOTHESIS
}

\author{
S. M. GONEK
}

\begin{abstract}
We investigate the approximation of the Riemann zeta-function by short truncations of its Euler product in the critical strip. We then construct a parameterized family of non-analytic functions that approximate the zetafunction to the right of the critical line. With the possible exception of finitely many zeros off the critical line, each function in the family satisfies a Riemann Hypothesis. Moreover, when the parameter is not too large, the functions in the family have about the same number of zeros as the zeta-function, their zeros are all simple, and the zeros "repel". The structure of these functions makes the reason for the simplicity and repulsion of their zeros apparent. Computer calculations suggest that the zeros of functions in the family are remarkably close to those of the zeta-function, even for small values of the parameter. We show that if the Riemann Hypothesis holds for the Riemann zeta-function, then the zeros of these functions indeed converge to those of the zeta-function as the parameter increases and that, between consecutive zeros of the zeta-function, the functions tend to twice the zeta-function. Finally, we discuss analogues of the model for other L-functions and the insight they give into the distribution of zeros of linear combinations of L-functions.
\end{abstract}

\section{Contents}

1. Introduction

2. The approximation of $\zeta(s)$ by finite Dirichlet series

3. The approximation of $\zeta(s)$ by finite Euler products

4. Products, sums, and moments

5. A function related to the zeta-function

6. The Riemann Hypothesis for $\zeta_{X}(s)$

7. The number of zeros of $\zeta_{X}(s)$

8. The number of simple zeros of $\zeta_{X}(s)$

9. The relative sizes of $\zeta_{X}(s)$ and $\zeta(s)$ and the relation between their zeros

10. Why zeros of $\zeta_{X}(s)$ are simple and repel

11. Other L-functions and sums of L-functions

12. Appendix

Acknowledgements

Received by the editors September 17, 2010.

2010 Mathematics Subject Classification. Primary 11M06, 11M26.

This work was supported in part by NSF grant DMS-0653809.

(C)2011 American Mathematical Society Reverts to public domain 28 years from publication 


\section{INTRODUCTION}

Why should the Riemann Hypothesis be true? If all the zeros of the zeta-function are simple, then why? Why do the zeros seem to repel each other? We know that an eventual proof of the Riemann Hypothesis must use, at least implicitly, both the zeta-function's Euler product and functional equation. For there are functions with similar functional equations but no Euler product and functions with an Euler product but no functional equation, for which the Riemann Hypothesis is false. But why are these two ingredients essential? This paper began as an attempt to gain insight into these questions.

Section 2 begins with a brief discussion of the approximation of $\zeta(s)$ by truncations of its Dirichlet series. In Section 3 we turn to the approximation of $\zeta(s)$ by truncations of its Euler product. We show that if the Riemann Hypothesis is true, then short products approximate the zeta-function well in a region containing most of the right-half of the critical strip. Conversely, a good approximation by products in this region implies the zeta-function has at most finitely many zeros there. Section 4 is a slight departure from the main direction of the paper, but we include it in order to deduce some immediate consequences of the results of Section 3. In Section 5 we construct a parameterized family, $\left\{\zeta_{X}(s)\right\}$, of functions related to the zeta-function with the same type of approximation property as the finite Euler products. That is, if the Riemann Hypothesis is true, then $\zeta_{X}(s)$ is a good approximation of $\zeta(s)$ in a certain region in the right-half of the critical strip and, if $\zeta_{X}(s)$ approximates $\zeta(s)$ well in this region, then $\zeta(s)$ can have at most finitely many zeros there. In Section 6 we show that, with the possible exception of a few zeros near the real axis, a Riemann Hypothesis holds for each $\zeta_{X}(s)$. In Sections 7 and 8 we prove that on the Riemann Hypothesis, if the parameter $X$ is not too large, then $\zeta_{X}(s)$ has about the same number of zeros as $\zeta(s)$ and that its zeros are all simple (again with the possible exception of a few low lying ones). We also show unconditionally that when the parameter is much larger, $\zeta_{X}(s)$ still has asymptotically the same number of zeros as $\zeta(s)$ and that $100 \%$ of these (in the density sense) are simple. In the next section we study relationships between the two functions on the critical line. Assuming the Riemannn Hypothesis, we show that the zeros of $\zeta_{X}(s)$ converge to the zeros of $\zeta(s)$ as $X \rightarrow \infty$ and that between the zeros of the zeta-function $\zeta_{X}\left(\frac{1}{2}+i t\right) \rightarrow 2 \zeta\left(\frac{1}{2}+i t\right)$. In Section 10 we examine the structural source of the simplicity and repulsion of the zeros of $\zeta_{X}(s)$ and discuss whether this sheds any light on the analogous properties of the zeros of $\zeta(s)$. In the last section we illustrate how our results generalize to other L-functions by defining functions $L_{X}(s, \chi)$ corresponding to the Dirichlet L-function $L(s, \chi)$. We then study the distribution of zeros of linear combinations of $L_{X}(s, \chi)$. This suggests a heuristic different from the usual one (of carrier waves) for understanding why linear combinations of the standard L-functions should have $100 \%$ of their zeros on the critical line. The Appendix provides some useful approximations of the zeta-function by Dirichlet polynomials.

The functions $\zeta_{X}(s)$ are simpler than the Riemann zeta-function yet they capture some of its most important structural features. It is therefore reasonable to regard them as "models" of the zeta-function. The modeling is best when $X$ is large, say a power of $t$, but so far, with the exception of Theorem 9.1 our results are most satisfactory only for smaller $X$ ranges.

We began by raising several deep questions. Although we do not offer definitive answers, we hope our investigation will be seen as suggestive. For example, a 
possible interpretation of our results as they touch on the question of why both the Euler product and functional equation are necessary for the truth of the Riemann Hypothesis is this: the Euler product prevents the zeta-function from having zeros off the line, while the functional equation puts them on it.

\section{The approximation of $\zeta(s)$ By finite Dirichlet Series}

Throughout we write $s=\sigma+i t$ and $\tau=|t|+2 ; \epsilon$ denotes an arbitrarily small positive number which may not be the same at each occurrence.

The Riemann zeta-function is analytic in the entire complex plane, except for a simple pole at $s=1$, and in the half-plane $\sigma>1$ it is given by the absolutely convergent series

$$
\zeta(s)=\sum_{n=1}^{\infty} n^{-s} .
$$

Estimating the tail of the series trivially, we obtain the approximation

$$
\zeta(s)=\sum_{n=1}^{X} n^{-s}+O\left(\frac{X^{1-\sigma}}{\sigma-1}\right)
$$

for $\sigma>1$ and $X \geq 1$. A crude form of the approximate functional equation (see Titchmarsh [25]) extends this into the critical strip:

$$
\zeta(s)=\sum_{n=1}^{X} n^{-s}+\frac{X^{1-s}}{s-1}+O\left(X^{-\sigma}\right) .
$$

This holds uniformly for $\sigma \geq \sigma_{0}>0$, provided that $X \geq C \tau / 2 \pi$, where $C$ is any constant greater than 1 . The second term on the right-hand side reflects the simple pole of $\zeta(s)$ at $s=1$ and, if we stay away from it, it can be ignored. For instance, setting $X=t$ and assuming $t \geq 1$, we find that

$$
\zeta(s)=\sum_{n \leq t} n^{-s}+O\left(t^{-\sigma}\right)
$$

uniformly for $\sigma \geq \sigma_{0}>0$. Thus, truncations of the Dirichlet series defining $\zeta(s)$ approximate it well, even in the critical strip.

Now suppose that the Lindelöf Hypothesis is true. That is,

$$
\zeta\left(\frac{1}{2}+\mathrm{i} t\right) \ll \tau^{\epsilon} .
$$

Then the length of the series in (2.2) and (2.3) may be reduced considerably, as the following modification of Theorem 13.3 of Titchmarsh [25] shows (see the Appendix for a proof).

Theorem 2.1. Let $\sigma$ be bounded, $|\sigma| \geq \frac{1}{2}$, and $|s-1|>\frac{1}{10}$. Also let $1 \leq X \leq t^{2}$. A necessary and sufficient condition for the truth of the Lindelof Hypothesis is that

$$
\zeta(s)=\sum_{n \leq X} n^{-s}+O\left(X^{\frac{1}{2}-\sigma} \tau^{\epsilon}\right) .
$$

It follows from this that if the Lindelöf Hypothesis is true and we stay away from the pole of the zeta-function at $s=1$, then $\zeta(s)$ is well-approximable by arbitrarily short truncations of its Dirichlet series in the half plane $\sigma>\frac{1}{2}$. As we have seen, this is unconditionally true in the half plane $\sigma>1$. 
On the other hand, short sums cannot approximate $\zeta(s)$ well in the strip $0<$ $\sigma \leq 1 / 2$. Suppose that such a sum and $\zeta(s)$ were within $\epsilon$ of each other, where $\epsilon>0$ is small. Then we would have

$$
\int_{T}^{2 T}\left|\zeta(s)-\sum_{n \leq X} n^{-s}\right|^{2} d t \leq \epsilon^{2} T .
$$

However, if $0<\sigma \leq \frac{1}{2}$ is fixed and $X<T^{1-\epsilon}$, then

$$
\int_{T}^{2 T}\left|\sum_{n \leq X} n^{-s}\right|^{2} d t \sim \begin{cases}T\left(\frac{X^{1-\sigma}-1}{1-\sigma}\right) & \text { if } \sigma<\frac{1}{2} \\ T \log X & \text { if } \sigma=\frac{1}{2}\end{cases}
$$

and

$$
\int_{T}^{2 T}|\zeta(\sigma+i t)|^{2} d t \sim \begin{cases}C(\sigma) T^{2-2 \sigma} & \text { if } \sigma<\frac{1}{2}, \\ T \log T & \text { if } \sigma=\frac{1}{2} .\end{cases}
$$

Comparing these when $\sigma<\frac{1}{2}$ and again when $\sigma=\frac{1}{2}$, we obtain a contradiction to (2.4). This argument is unconditional and shows that one cannot do better than (2.3) even if the Lindelöf or Riemann Hypothesis is true.

To summarize, $\zeta(s)$ is well-approximable unconditionally by arbitrarily short truncations of its Dirichlet series in the region $\sigma>1,|s-1|>\frac{1}{10}$. On the Lindelöf Hypothesis this remains true even in the right-half of the critical strip, $\frac{1}{2}<\sigma \leq 1$. However, on and to the left of the critical line, the length of the truncation must $\mathrm{be} \approx t$. The situation is the same if we assume the Riemann Hypothesis instead of the Lindelöf Hypothesis, since the former implies the latter.

\section{The approximation of $\zeta(s)$ By finite Euler products}

The zeta-function also has the Euler product representation

$$
\zeta(s)=\prod_{p}\left(1-p^{-s}\right)^{-1}
$$

in the half-plane $\sigma>1$, where the product is over all prime numbers. This converges absolutely, and it is straightforward to show (take logarithms) that

$$
\zeta(s)=\prod_{p \leq X}\left(1-p^{-s}\right)^{-1}\left(1+O\left(\frac{X^{1-\sigma}}{(\sigma-1) \log X}\right)\right),
$$

for $\sigma>1$. Here we implicitly use the fact that $\zeta(s)$ does not vanish in $\sigma>1$. As is often the case, it is more natural from an analytic point of view to work with weighted approximations, so we will use expressions of the type

$$
\exp \left(\sum_{n} \frac{\Lambda(n) v(n)}{n^{s} \log n}\right)
$$

where $\Lambda(n)$ is von Mangoldt's function and the weights $v(n)$ will be specified later.

We next ask whether it is possible to extend (3.1) or a weighted form of it into the critical strip in the same way that (2.2) extended (2.1). The following recent result of Gonek, Hughes, and Keating [10] suggests an answer.

Theorem 3.1. Let $s=\sigma+i t$ with $\sigma \geq 0$ and $|t| \geq 2$, let $X \geq 2$ be a real parameter, and let $K$ be any fixed positive integer. Let $f(x)$ be a non-negative $C^{\infty}$ function 
of mass 1 supported on $[0,1]$ and set $u(x)=X f\left(X \log \frac{x}{e}+1\right) / x$ (so that $u(x)$ is supported on $\left.\left[e^{1-1 / X}, e\right]\right)$. Set

$$
U(z)=\int_{0}^{\infty} u(x) E_{1}(z \log x) d x
$$

where $E_{1}(z)$ is the exponential integral $\int_{z}^{\infty} e^{-w} / w d w$. Then

$$
\zeta(s)=P_{X}(s) Z_{X}(s)\left(1+O\left(\frac{X^{K+2}}{(|s| \log X)^{K}}\right)+O\left(X^{-\sigma} \log X\right)\right),
$$

where

$$
P_{X}(s)=\exp \left(\sum_{n \leq X} \frac{\Lambda(n)}{n^{s} \log n}\right)
$$

$\Lambda(n)$ is von Mangoldt's function, and

$$
Z_{X}(s)=\exp \left(-\sum_{\rho_{n}} U\left(\left(s-\rho_{n}\right) \log X\right)\right)
$$

The constants implied by the $O$-terms depend only on $f$ and $K$.

Thus, if $X<|t|^{1-3 /(K+2)}$, for example, then $\zeta(s)$ factors in the region $\sigma \geq 0$, $|t| \geq 2$ as

$$
\zeta(s)=\exp \left(\sum_{n \leq X} \frac{\Lambda(n)}{n^{s} \log n}\right) Z_{X}(s)(1+o(1)),
$$

where $Z_{X}(s)$ is a product over the zeros of $\zeta(s)$. Now one can show that, in the right-half of the critical strip, $Z_{X}(s)$ is close to 1 as long as $s$ is not too near a zero of $\zeta(s)$. Hence, if the Riemann Hypothesis is true and $s$ is not too close to the critical line, $Z_{X}(s)$ will be close to 1 . (The closer $\sigma$ is to $\frac{1}{2}$, the larger one needs to take $X$.) Thus, under the Riemann Hypothesis, an analogue of (3.1) does hold in the right-half of the critical strip.

To prove these assertions it is convenient to work with an alternative form of (3.2), and we derive this next.

Write

$$
P_{X}(s)=\exp \left(\sum_{n \leq X^{2}} \frac{\Lambda_{X}(n)}{n^{s} \log n}\right),
$$

where

$$
\Lambda_{X}(n)= \begin{cases}\Lambda(n) & \text { if } n \leq X \\ \Lambda(n)\left(2-\frac{\log n}{\log X}\right) & \text { if } X<n \leq X^{2} \\ 0 & \text { if } n>X^{2}\end{cases}
$$

Also, let

$$
E_{2}(z)=\int_{z}^{\infty} \frac{e^{-w}}{w^{2}} d w \quad(z \neq 0)
$$

denote the second exponential integral, and set

$$
F_{2}(z)=2 E_{2}(2 z)-E_{2}(z) .
$$


Finally, define

$$
Z_{X}(s)=\exp \left(\sum_{\rho} F_{2}((s-\rho) \log X)-F_{2}((s-1) \log X)\right),
$$

where the sum is over all the non-trivial zeros, $\rho=\beta+i \gamma$, of the zeta-function. Our modified version of (3.2) is

Theorem 3.2. Let $\sigma \geq 0$ and $X \geq 2$. With $P_{X}(s)$ and $Z_{X}(s)$ as above we have

$$
\zeta(s)=P_{X}(s) Z_{X}(s)\left(1+O\left(\frac{X^{-\sigma-2}}{\tau^{2} \log ^{2} X}\right)\right) .
$$

Proof. We begin with the explicit formula (see Titchmarsh [25], Theorem 14.20)

$$
\begin{gathered}
\frac{\zeta^{\prime}}{\zeta}(s)=-\sum_{n \leq X^{2}} \frac{\Lambda_{X}(n)}{n^{s}}+\frac{X^{2(1-s)}-X^{(1-s)}}{(1-s)^{2} \log X}+\sum_{\rho} \frac{X^{\rho-s}-X^{2(\rho-s)}}{(s-\rho)^{2} \log X} \\
+\frac{1}{\log X} \sum_{q=1}^{\infty} \frac{X^{-(2 q+s)}-X^{-2(2 q+s)}}{(s+2 q)^{2}} .
\end{gathered}
$$

The last term on the right is easily seen to be $\ll X^{-\sigma-2} / \tau^{2} \log X$, so we have

$$
\frac{\zeta^{\prime}}{\zeta}(s)=-\sum_{n \leq X^{2}} \frac{\Lambda_{X}(n)}{n^{s}}+\frac{X^{2(1-s)}-X^{(1-s)}}{(1-s)^{2} \log X}+\sum_{\rho} \frac{X^{\rho-s}-X^{2(\rho-s)}}{(s-\rho)^{2} \log X}+O\left(\frac{X^{-\sigma-2}}{\tau^{2} \log X}\right) .
$$

Next we integrate (3.8) from $\infty$ to $s_{0}=\sigma_{0}+i t$, where $\sigma_{0}+i t$ is not a zero of the zeta-function. We use the convention that if $t$ is the ordinate of a zero $\rho=\beta+i \gamma$ and $0 \leq \sigma_{0}<\beta$, then

$$
\log \zeta\left(\sigma_{0}+i \gamma\right)=\lim _{\epsilon \rightarrow 0^{+}} \log \zeta\left(\sigma_{0}+i(\gamma+\epsilon)\right) .
$$

The $O$-term contributes

$$
\ll \frac{X^{-\sigma_{0}-2}}{\tau^{2} \log ^{2} X} .
$$

We also see that

$$
\int_{\infty}^{\sigma_{0}+i t} \frac{X^{\rho-s}-X^{2(\rho-s)}}{(s-\rho)^{2}} d s=\log X F_{2}\left(\left(s_{0}-\rho\right) \log X\right) .
$$

Here we use the convention analogous to (3.9) if $t$ is the ordinate of a zero. It follows that

$$
\begin{aligned}
\log \zeta\left(s_{0}\right)= & \sum_{n \leq X^{2}} \frac{\Lambda_{X}(n)}{n^{s_{0}} \log n}+\sum_{\rho} F_{2}\left(\left(s_{0}-\rho\right) \log X\right)-F_{2}\left(\left(s_{0}-1\right) \log X\right) \\
& +O\left(\frac{X^{-\sigma_{0}-2}}{\tau^{2} \log ^{2} X}\right) .
\end{aligned}
$$

Replacing $\sigma_{0}$ by $\sigma$ and $s_{0}$ by $s$ and exponentiating both sides, we obtain the stated result when $s$ is not equal to a zero $\rho$. If it is, we may interpret the factor in (3.6) corresponding to $\rho$ as $\lim _{\epsilon \rightarrow 0^{+}} \exp \left(F_{2}(i \epsilon \log X)\right)$. From the well-known formula $E_{2}(z)=1 / z+\log z+e_{2}(z)$, where $|\arg z|<\pi$ and $e_{2}(z)$ is analytic in $z$, it follows that

$$
F_{2}(z)=\log 4 z+f_{2}(z) \quad(|\arg z|<\pi),
$$


where $f_{2}(z)$ is also analytic. Therefore $\lim _{\epsilon \rightarrow 0^{+}} \exp \left(F_{2}(i \epsilon \log X)\right)=0$. Since this agrees with the left-hand side of (3.7), the formula is valid in this case as well.

Before stating our next result we require some notation and a lemma. As usual we write $S(t)=(1 / \pi) \arg \zeta\left(\frac{1}{2}+i t\right)$ with the convention that if $t$ is the ordinate of a zero, $S(t)=\lim _{\epsilon \rightarrow 0^{+}} S(t+\epsilon)$. For $t \geq 0$ we let $\Phi(t)$ denote a positive increasing differentiable function such that

$$
|S(t)| \leq \Phi(t) \quad \text { and } \quad\left|\zeta\left(\frac{1}{2}+i t\right)\right| \ll \exp (\Phi(t)),
$$

and such that for $t$ sufficiently large we have

$$
\Phi^{\prime}(t) / \Phi(t) \ll \frac{1}{t \log t} .
$$

We call such a function admissible. Any function of the type $(\log \tau)^{\alpha}(\log \log 3 \tau)^{\beta}$ with $\alpha$ positive satisfies (3.12). Furthermore, it is easy to show that if $\Phi$ satisfies (3.12), then

$$
\Phi\left(t^{a}\right) \ll \Phi(t),
$$

where the implied constant depends at most on $a$. It is known that $\Phi(t)=\frac{1}{6} \log \tau$ is admissible and, on the Lindelöf Hypothesis, that $\epsilon \log \tau$ is for any $\epsilon>0$. If the Riemannn Hypothesis is true, then $\Phi(t)=\frac{1}{2} \log \tau / \log \log 2 \tau$ is admissible. (The constant $\frac{1}{2}$ is a recent result due to Goldston and Gonek [8].) Balasubramaian and Ramachandra [5] (see also Titchmarsh [25], pp. 208-209 and p. 384) have shown that if $\Phi$ is admissible, $\Phi(t)=\Omega(\sqrt{\log \tau / \log \log 2 \tau})$, and this is unconditional. Farmer, Gonek and Hughes [7] have conjectured that $\Phi(t)=\sqrt{\left(\frac{1}{2}+\epsilon\right) \log \tau \log \log 2 \tau}$ is admissible, but $\Phi(t)=\sqrt{\left(\frac{1}{2}-\epsilon\right) \log \tau \log \log 2 \tau}$ is not.

For the remainder of this paper $\Phi$ will always denote an admissible function.

We can now state our lemma.

Lemma 3.3. Assume the Riemann Hypothesis. Suppose that $\Phi(t)$ is admissible and that $\sigma>\frac{1}{2}$ is bounded. Then we have

$$
\sum_{\gamma} \frac{\sigma-\frac{1}{2}}{\left(\sigma-\frac{1}{2}\right)^{2}+(t-\gamma)^{2}} \ll \log \tau+\frac{\Phi(t)}{\sigma-\frac{1}{2}} .
$$

Moreover, if $\Delta>0$, then

$$
\sum_{|\gamma-t|>\Delta} \frac{1}{(t-\gamma)^{2}} \ll \frac{1}{\Delta}\left(\log \tau+\frac{\Phi(\tau)}{\Delta}\right) .
$$

Remark. In fact one can show that the first sum equals $\frac{1}{2} \log \tau+O\left(\Phi(\tau) /\left(\sigma-\frac{1}{2}\right)\right)$, but we do not require this.

Proof. For the sake of convenience we write $\sigma-\frac{1}{2}=a$. Recall that $N(t)$, the number of zeros of $\zeta(s)$ with ordinates in $[0, t]$, is

$$
N(t)=\frac{t}{2 \pi} \log \frac{t}{2 \pi}-\frac{t}{2 \pi}+\frac{7}{8}+S(t)+O\left(\frac{1}{\tau}\right) .
$$

Therefore

$$
N(t+a)-N(t-a)=\frac{a}{\pi} \log \frac{t}{2 \pi}+O(\Phi(\tau))
$$


The left-hand side of (3.14) is

$$
\ll \sum_{0 \leq k \leq 1 / a}\left(\sum_{k a \leq|\gamma-t| \leq(k+1) a} \frac{a}{a^{2}+(t-\gamma)^{2}}\right)+\sum_{|\gamma-t|>1} \frac{a}{a^{2}+(t-\gamma)^{2}} .
$$

Using (3.16), we see that the second sum is $\ll a \log \tau$, and that for each $k$ the sum in parentheses is

$$
\ll(a \log \tau+\Phi(\tau)) \frac{a}{a^{2}+(k a)^{2}} \ll \frac{1}{1+k^{2}}\left(\log \tau+\frac{\Phi(\tau)}{a}\right) .
$$

Summing these estimates, we obtain (3.14).

The proof of (3.15) is similar.

Our approximation of the zeta-function by finite Euler products will follow almost immediately from

Theorem 3.4. Assume the Riemann Hypothesis. Let $\sigma \geq \frac{1}{2}+\frac{1}{\log X}$ and $|s-1| \geq \frac{1}{10}$. Then for any $X \geq 2$ we have

$$
\zeta(s)=P_{X}(s) e^{R_{X}(s)}
$$

where

$$
R_{X}(s) \ll X^{\frac{1}{2}-\sigma}\left(\Phi(\tau)+\frac{\log \tau}{\log X}\right)+\frac{X}{\tau^{2} \log ^{2} X} .
$$

Moreover, throughout the region $\sigma \geq \frac{1}{2},|s-1| \geq \frac{1}{10}$, and

$$
\arg \zeta(\sigma+i t)=-\sum_{n \leq X^{2}} \frac{\Lambda_{X}(n) \sin (t \log n)}{n^{\sigma} \log n}+O\left(R_{X}(s)\right) .
$$

Proof. We estimate

$$
Z_{X}(s)=\exp \left(\sum_{\rho} F_{2}((s-\rho) \log X)-F_{2}((s-1) \log X)\right)
$$

in (3.6).

Integrating (3.5) by parts, we see that for $|z| \geq 1$,

$$
E_{2}(z)=\frac{e^{-z}}{z^{2}}\left(1+O\left(|z|^{-1}\right)\right)
$$

and therefore that

$$
F_{2}(z) \ll e^{\max (-\operatorname{Re} z,-\operatorname{Re} 2 z)} /|z|^{2} .
$$

Since $\sigma \geq \frac{1}{2}+\frac{1}{\log X}$ and the zeros are of the form $\rho=\frac{1}{2}+i \gamma$, they all satisfy $|s-\rho| \log X \geq 1$. Thus, by (3.22) and Lemma 3.3, the sum in (3.20) is

$$
\ll \frac{1}{\log ^{2} X} \sum_{|s-\rho| \log X \geq 1} \frac{X^{\frac{1}{2}-\sigma}}{\left(\sigma-\frac{1}{2}\right)^{2}+(t-\gamma)^{2}} \ll X^{\frac{1}{2}-\sigma}\left(\Phi(\tau)+\frac{\log \tau}{\log X}\right) .
$$

Also by (3.22),

$$
F_{2}((s-1) \log X) \ll \frac{X^{\max (1-\sigma, 2(1-\sigma))}}{\tau^{2} \log ^{2} X} .
$$

The first assertion of the theorem follows from this and (3.7). 
The second assertion follows immediately from (3.17) if $\sigma \geq \frac{1}{2}+\frac{1}{\log X}$, so we need only consider the case $\frac{1}{2} \leq \sigma<\frac{1}{2}+\frac{1}{\log X}$. The terms in the sum of (3.20) for which $|s-\rho| \log X \geq 1$ contribute the same amount as before. However, now there may also be a finite number of terms for which $|s-\rho| \log X \leq 1$. Using (3.11) to estimate these, we find that if $s$ is not a zero, they contribute

$$
\sum_{|s-\rho| \log X \leq 1}(\log (4(s-\rho) \log X)+O(1)) .
$$

Since $|\arg (s-\rho) \log X| \leq \pi / 2$, the imaginary part of this is

$$
\ll \sum_{|t-\gamma| \leq 1 / \log X} 1 \ll \frac{\log \tau}{\log X}+\Phi(t),
$$

by (3.16). This is big- $O$ of the bound in (3.23) because $\frac{1}{2} \leq \sigma<\frac{1}{2}+\frac{1}{\log X}$. Thus, we obtain (3.19) provided that $t$ is not the ordinate of a zero. If it is, the result follows from our convention that $\arg \zeta(\sigma+i t)=\lim _{\epsilon \rightarrow 0^{+}} \arg \zeta(\sigma+i(t+\epsilon))$. This completes the proof of the theorem.

We can now deduce an approximation of $\zeta(s)$ by Euler products.

Theorem 3.5. Assume the Riemann Hypothesis. Let $|s-1| \geq \frac{1}{10}$ and $\exp (\log \tau / \Phi(t))$ $\leq X \leq \tau^{2}$. Then if $\frac{1}{2}+\frac{C \log \Phi(t)}{\log X} \leq \sigma \leq 1$ with $C>1$, we have

$$
\zeta(s)=P_{X}(s)\left(1+O\left(\Phi(t)^{1-C}\right)\right) .
$$

If $2 \leq X<\exp (\log \tau / \Phi(t))$ and $\frac{1}{2}+\frac{C \log \log 2 \tau}{\log X} \leq \sigma \leq 1$ with $C>1$, then

$$
\zeta(s)=P_{X}(s)\left(1+O\left((\log \tau)^{1-C}\right)\right) .
$$

Proof. We estimate $R_{X}(s)$ in (3.17). First assume that $\exp (\log \tau / \Phi(t)) \leq X \leq \tau^{2}$ and $\frac{1}{2}+\frac{C \log \Phi(t)}{\log X} \leq \sigma \leq 1$. Then $\log \tau / \log X \leq \Phi(t)$ and

$$
\begin{aligned}
R_{X}(s) & \ll X^{\frac{1}{2}-\sigma} \Phi(t)+\frac{X^{2\left(\frac{1}{2}-\sigma\right)}}{\log ^{2} X} \\
& \ll \Phi(t)^{1-C}+\Phi(t)^{-2 C} \\
& \ll \Phi(t)^{1-C} .
\end{aligned}
$$

It follows that $\exp \left(R_{X}(s)\right)=1+O\left(\Phi(t)^{1-C}\right)$, so we have (3.25). The second assertion follows similarly, except that this time $\Phi(t)<\log \tau / \log X$.

Thus, on the Riemann Hypothesis, short Euler products approximate $\zeta(s)$ as long as we are not too close to the critical line.

We can combine the two assertions of Theorem 3.5 and prove a partial converse as well.

Theorem 3.6. Assume the Riemann Hypothesis. Let $2 \leq X \leq t^{2},|s-1| \geq \frac{1}{10}$, and $\frac{1}{2}+\frac{C \log \log 2 \tau}{\log X} \leq \sigma \leq 1$ with $C>1$. Then

$$
\zeta(s)=P_{X}(s)\left(1+O\left(\log ^{(1-C) / 2} t\right)\right) .
$$

Conversely, if (3.26) holds for $2 \leq X \leq t^{2}$ in the region stated, then $\zeta(s)$ has at most a finite number of zeros to the right of $\sigma=\frac{1}{2}+\frac{C \log \log 2 \tau}{\log X}$. 
Remark. The condition $\sigma \geq \frac{1}{2}+\frac{C \log \log 2 \tau}{\log X}$ implies a lower bound for $X$ that grows with $t$, namely,

$$
X \geq(\log \tau)^{C /\left(\sigma-\frac{1}{2}\right)} .
$$

The converse follows from the observation that if 3.26 holds, then there is a constant $B>0$ such that

$$
\left|\zeta(s) P_{X}(s)^{-1}-1\right| \leq B \log ^{(1-C) / 2} \tau .
$$

If $\zeta(\beta+i \gamma)=0$ with $\beta>\frac{1}{2}+\frac{C \log \log (2|\gamma|+2)}{\log X}$, this forces $\gamma \leq \exp \left(B^{2 /(C-1)}\right)$, and the result follows.

As in the case of approximations by short sums, one can also ask whether short products approximate $\zeta(s)$ well when $0<\sigma \leq \frac{1}{2}$. For sums we saw that the answer is no unless they are of length at least $t$. For products the answer is no, no matter how long they are. A quick way to see this is by counting zeros of $\zeta(s)$ and of $P_{X}(s)$ in a rectangle containing the segment $\left[\frac{1}{2}, \frac{1}{2}+i T\right]$. The former has $\sim(T / 2 \pi) \log T$ zeros, the latter none. This would be impossible if $\zeta(s)=P_{X}(s)(1+o(1))$ in the rectangle.

One can also argue as follows when $\sigma$ is strictly less than $\frac{1}{2}$. (A modification of the argument works for $\sigma=\frac{1}{2}$, too.) Suppose that $\zeta(s)=P_{X}(s)(1+o(1))$ in the strip $0<\sigma \leq \frac{1}{2}$. Then $\log |\zeta(s)|=\log \left|P_{X}(s)\right|+o(1)$, and we have

$$
\int_{0}^{T}(\log |\zeta(\sigma+i t)|)^{2} d t \sim \int_{0}^{T}\left(\log \left|P_{X}(\sigma+i t)\right|\right)^{2} d t
$$

for $\sigma$ fixed and $T \rightarrow \infty$. By the functional equation for the zeta-function,

$$
\log |\zeta(\sigma+i t)|=\left(\frac{1}{2}-\sigma\right) \log \frac{\tau}{2 \pi}+\log |\zeta(1-\sigma-i t)|+o(1) .
$$

Now the mean-square of the three terms on the right-hand side are $\sim\left(\frac{1}{2}-\sigma\right)^{2} T \log ^{2} T$, $\sim c_{0} T$, and $o(T)$, respectively. Thus,

$$
\int_{0}^{T}(\log |\zeta(\sigma+i t)|)^{2} d t \sim\left(\frac{1}{2}-\sigma\right)^{2} T \log ^{2} T .
$$

On the other hand, by the mean value theorem for Dirichlet polynomials, if $X=$ $o\left(T^{\frac{1}{2}}\right)$, the right-hand side of (3.27) is

$$
\begin{aligned}
\int_{0}^{T}\left(\log \left|P_{X}(\sigma+i t)\right|\right)^{2} d t & \sim \int_{0}^{T}\left(\sum_{n \leq X^{2}} \frac{\Lambda_{X}(n) \cos (t \log n)}{n^{\sigma} \log n}\right)^{2} d t \\
& \sim \frac{T}{2} \sum_{n \leq X^{2}} \frac{\Lambda_{X}^{2}(n)}{n^{2 \sigma} \log ^{2} n} \\
& \sim c T \frac{X^{2-4 \sigma}}{\log X}
\end{aligned}
$$

where $c$ is a positive constant. Comparing this with (3.28), we see that (3.27) cannot hold if $0 \leq \sigma<\frac{1}{2}$ and $X$ is larger than a certain power of $\log T$. Note also that for infinitely many $t$ tending to infinity, $P_{X}(s)$ can be quite large, namely

$$
\left|P_{X}(\sigma+i t)\right| \gg \exp \left(X^{1-2 \sigma} / \sqrt{\log X}\right) .
$$

In this section we have seen that short truncations of its Euler product approximate $\zeta(s)$ well in the region $\sigma>1,|s-1|>\frac{1}{10}$. We also showed that this remains 
true in the right-half of the critical strip if the Riemann Hypothesis is true and if we are not too near the critical line (and use a weighted Euler product). However, to the left of the critical line the Euler product is not a good approximation of $\zeta(s)$, regardless of how long it is.

\section{Products, sums, and moments}

Our purpose in this section is to deduce two consequences of the results of the previous section. First we require a result whose proof is given in the Appendix.

Theorem 4.1. Assume the Riemann Hypothesis. Let $\sigma \geq \frac{1}{2}$ be bounded, $|s-1|>$ $\frac{1}{10}$, and $2 \leq X \leq t^{2}$. Then there is a positive constant $C_{1}$ such that

$$
\zeta(s)=\sum_{n \leq X} n^{-s}+O\left(X^{\frac{1}{2}-\sigma} e^{C_{1} \Phi(t)}\right) .
$$

If $\frac{1}{2}+2 C_{1} \frac{\Phi(t)}{\log X} \leq \sigma \leq 1$, the error term in Theorem 4.1 is $O\left(e^{-C_{1} \Phi(t)}\right)$. For the same $\sigma$-range, the approximation of $\zeta(s)$ given by Theorem 3.4 is

$$
\zeta(s)=P_{X}(s)\left(1+O\left(e^{-(2-\epsilon) C_{1} \Phi(t)}\right)\right),
$$

where $\epsilon$ is arbitrarily small. Thus, equating respective sides of (4.1) and (4.2) and solving for $P_{X}(s)$, we see that

$$
P_{X}(s)=\left(\sum_{n \leq X} \frac{1}{n^{s}}+O\left(e^{-2 C_{1} \Phi(t)} g\right)\right)\left(1+O\left(e^{-(2-\epsilon) C_{1} \Phi(t)}\right)\right) .
$$

By the corollary to Theorem 4.1 (see the Appendix), the sum here is $\ll e^{C_{1} \Phi(t)}$, so we obtain

$$
P_{X}(s)=\sum_{n \leq X} \frac{1}{n^{s}}+O\left(e^{-(1-\epsilon) C_{1} \Phi(t)}\right) .
$$

We have now proved

Theorem 4.2. Assume the Riemann Hypothesis. Let $\sigma \geq \frac{1}{2}$ be bounded, $|s-1|>$ $\frac{1}{10}$, and $2 \leq X \leq t^{2}$. There is a positive constant $C_{1}$ such that if $\frac{1}{2}+\frac{2 C_{1} \Phi(t)}{\log X} \leq \sigma \leq 1$, then

$$
\sum_{n \leq X} \frac{1}{n^{s}}=P_{X}(s)+O\left(e^{-C_{2} \Phi(t)}\right)
$$

for any positive constant $C_{2}$ less than $C_{1}$.

Our second observation is that one can use these appoximations to calculate the moments of a very long Euler product. Suppose one wished to compute the moments

$$
\int_{0}^{T}\left|P_{X}(\sigma+i t)\right|^{2 k} d t
$$

when $\frac{1}{2}<\sigma<1$. The standard method would be to write $P_{X}(s)^{k}$ as a Dirichlet series and use a mean value theorem for such polynomials to compute the mean modulus squared. But this only works well when the product does not have many factors. For example, for a slightly different Euler product, Gonek, Hughes and Keating [10] have proved the following. 
Theorem. Let $1 / 2<c<1, \epsilon>0$, and let $k$ be any positive real number. Suppose that $X$ and $T \rightarrow \infty$ and $X=O\left((\log T)^{1 /(1-c+\epsilon)}\right)$. Then we have

$$
\int_{T}^{2 T}\left|\exp \left(\sum_{n \leq X} \frac{\Lambda(n)}{n^{\sigma+i t} \log n}\right)\right|^{2 k} d t \sim a_{k}(\sigma) T \zeta(2 \sigma)^{k^{2}} e^{-k^{2} E_{1}((2 \sigma-1) \log X)},
$$

where

$$
a_{k}(\sigma)=\prod_{p}\left(\left(1-\frac{1}{p^{2 \sigma}}\right)^{k^{2}} \sum_{m=0}^{\infty} \frac{d_{k}\left(p^{m}\right)^{2}}{p^{2 m \sigma}}\right)
$$

uniformly for $c \leq \sigma \leq 1$. Here $d_{k}(n)$ is the $k$ th divisor function and $E_{1}(z)=$ $\int_{z}^{\infty} \frac{e^{-w}}{w} d w$ is the first exponential integral.

Note that here the number of factors in the Euler product is not even $\log ^{2} T$. On the other hand, if we assume the Riemann Hypothesis, that $\frac{1}{2}+\frac{C \log \log 2 T}{\log X} \leq \sigma<1$ with $C>1$, and that $2 \leq X \leq T^{2}$, then by Theorem 3.5

$$
\zeta(s)=P_{X}(s)(1+o(1)) \text {. }
$$

Hence,

$$
\int_{T}^{2 T}\left|P_{X}(\sigma+i t)\right|^{2 k} d t \sim \int_{T}^{2 T}|\zeta(\sigma+i t)|^{2 k} d t .
$$

Now, it is a consequence of the Lindelöf Hypothesis (Titchmarsh 25], Theorem 13.2), and so also of the Riemann Hypothesis, that when $\frac{1}{2}<\sigma<1$ is fixed,

$$
\int_{T}^{2 T}|\zeta(\sigma+i t)|^{2 k} d t \sim T \sum_{n=1}^{\infty} \frac{d_{k}^{2}(n)}{n^{2 \sigma}}
$$

for any fixed positive integer $k$. Thus, for such $\sigma$ and $k$ we have

$$
\int_{T}^{2 T}\left|P_{X}(\sigma+i t)\right|^{2 k} d t \sim T \sum_{n=1}^{\infty} \frac{d_{k}^{2}(n)}{n^{2 \sigma}} .
$$

This gives an estimation of the moments of an extremely long Euler product deep into the critical strip.

\section{A FUnCtion RELATED to The Zeta-FunCtion}

In Section 2 we showed that short truncations of its Dirichlet series approximate $\zeta(s)$ in $\sigma>1$ and that, if the Lindelöf Hypothesis is true, this also holds in $\sigma>\frac{1}{2}$. The approximation cannot be good in the strip $0<\sigma \leq \frac{1}{2}$ unless the length of the sum is of order at least $t$; and this is so even if we assume the Lindelöf or Riemann Hypothesis. In Section 3 we showed that the situation is similar, up to a point, when we approximate $\zeta(s)$ by the weighted Euler product $P_{X}(s)$ : short products approximate $\zeta(s)$ well in the half-plane $\sigma>1$ unconditionally, and in the strip $\frac{1}{2}<\sigma \leq 1$ on the Riemann Hypothesis. However, the approximation cannot be close in $0<\sigma<\frac{1}{2}$ no matter how many factors there are, for $P_{X}(s)$ gets much larger than $\zeta(s)$ in this strip (see (3.29)). We now re-examine the approximation of $\zeta(s)$ by sums when $\sigma$ is close to $\frac{1}{2}$. If we assume the Riemann Hypothesis, then by (4.1)

$$
\zeta(s)=\sum_{n \leq X} \frac{1}{n^{s}}+O\left(e^{-C_{1} \Phi(t)}\right)
$$


for $\frac{1}{2}+\frac{2 C_{1} \Phi(t)}{\log X} \leq \sigma \leq 1$ and $2 \leq X \leq t^{2}$. This is good for $X$ a small power of $t$ as long as $\sigma$ is not too close to $\frac{1}{2}$, but we know $X$ has to be of order $t$ on $\sigma=\frac{1}{2}$. This means the approximation is off by about $\sum_{X<n \leq t} n^{-s}$. The Hardy-Littlewood approximate functional equation [13] (or see Titchmarsh 25]) gives us another way to express this. It says that

$$
\zeta(s)=\sum_{n \leq X} \frac{1}{n^{s}}+\chi(s) \sum_{n \leq|t| / 2 \pi X} \frac{1}{n^{1-s}}+O\left(X^{-\sigma}\right)+O\left(\tau^{-\frac{1}{2}} X^{1-\sigma}\right),
$$

where $0 \leq \sigma \leq 1,|s-1| \geq \frac{1}{10}$, and $\chi(s)$ is the factor in the functional equation

$$
\zeta(s)=\chi(s) \zeta(1-s) .
$$

From this we see that the amount by which the sum $\sum_{n \leq X} n^{-s}$ is off from $\zeta(s)$ is about

$$
\chi(s) \sum_{n \leq|t| / 2 \pi X} \frac{1}{n^{1-s}} .
$$

If we let $X=\sqrt{|t| / 2 \pi}$ and note that $\left|\chi\left(\frac{1}{2}+i t\right)\right|=1$, we find that on the critical line $\zeta(s)$ is essentially composed of two pieces of equal size:

$$
\zeta\left(\frac{1}{2}+i t\right)=\sum_{n \leq X} \frac{1}{n^{\frac{1}{2}+i t}}+\chi\left(\frac{1}{2}+i t\right) \sum_{n \leq X} \frac{1}{n^{\frac{1}{2}-i t}}+O\left(\tau^{-\frac{1}{4}}\right) .
$$

In the case of Euler products, Theorem 3.5 suggests that $P_{X}(s)$ approximates $\zeta(s)$ well even closer to the critical line than a sum of length $X$ does. $P_{X}(s)$ is a good approximation when $\sigma \geq \frac{1}{2}+\frac{C \log \log 2 \tau}{\log X}$, while the sum is only close (as far as we know) when $\sigma \geq \frac{1}{2}+\frac{2 C_{1} \Phi(t)}{\log X}$. In light of this and (5.1) it is tempting to guess that

$$
\zeta(s) \approx P_{X}(s)+\chi(s) P_{X}(1-s),
$$

for some unspecified $X$. However, this is not a good guess. We have seen that $P_{X}(1-s)$ gets as large as $\exp \left(X^{\sigma-\frac{1}{2}} / \log X\right)$ when $\sigma>\frac{1}{2}$, whereas $\sum_{n \leq X} n^{s-1}$ is no larger than $X^{\sigma-\frac{1}{2}} e^{C_{1} \Phi(t)}$ by the Corollary in the Appendix.

The difficulty here is crossing the line $\sigma=\frac{1}{2}$, where there is a qualitative change in the behavior of the zeta-function. A way around this is to use the fact that the functional equation tells us the zeta-function everywhere once we know it in $\sigma \geq \frac{1}{2}$. If we restrict our attention to this half-plane, a reasonable alternative to (5.2) is

$$
\zeta_{X}(s)=P_{X}(s)+\chi(s) P_{X}(\bar{s}) .
$$

Note that on the critical line, $\zeta_{X}(s)$ and the right-hand side of (5.2) are identical. Also, since $\chi(s) \ll t^{\frac{1}{2}-\sigma}$ for $\sigma>\frac{1}{2}$ (see (6.1) below), we have

$$
\zeta_{X}(s)=P_{X}(s)\left(1+O\left(t^{\frac{1}{2}-\sigma}\right)\right) \text {. }
$$

Combining this observation with Theorem 3.6 , we obtain

Theorem 5.1. Assume the Riemann Hypothesis. Let $2 \leq X \leq t^{2},|s-1| \geq \frac{1}{10}$, and $\frac{1}{2}+\frac{C \log \log 2 \tau}{\log X} \leq \sigma \leq 1$ with $C>1$. Then

$$
\zeta(s)=\zeta_{X}(s)\left(1+O\left(\log ^{(1-C) / 2} \tau\right)\right) \text {. }
$$

Conversely, if (5.3) holds for $2 \leq X \leq t^{2}$ in the region stated, then $\zeta(s)$ has at most a finite number of zeros to the right of $\sigma=\frac{1}{2}+\frac{C \log \log 2 \tau}{\log X}$. 
Thus, even though $\zeta_{X}(s)$ is not analytic, it approximates $\zeta(s)$ well to the right of the critical line. It resembles the zeta-function closely in other ways too, as we shall see.

\section{The Riemann Hypothesis For $\zeta_{X}(s)$}

For a closer study of $\zeta_{X}(s)$ we require several properties of the chi-function

$$
\chi(s)=\pi^{s-\frac{1}{2}} \frac{\Gamma\left(\frac{1}{2}-\frac{1}{2} s\right)}{\Gamma\left(\frac{1}{2} s\right)},
$$

which appears in the functional equation of the zeta-function. Chi has simple poles at $s=1,3,5, \ldots$ from the $\Gamma$-factor in the numerator. If we stay away from these,

$$
\chi(s)=\left(\frac{\tau}{2 \pi}\right)^{\frac{1}{2}-\sigma-\mathrm{i} t} e^{\mathrm{i} t+\frac{1}{4} \mathrm{i} \pi}\left\{1+O\left(\frac{1}{\tau}\right)\right\}
$$

in any half-strip $-k<\sigma<k, t \geq 0$, by Stirling's approximation. When $t<0, \chi(s)$ is given by the conjugate of this. We note for later use that the $O$-term is infinitely differentiable.

Clearly $\left|\chi\left(\frac{1}{2}+i t\right)\right|=1$ for all $t$. The converse is also almost true.

Lemma 6.1. There is a positive absolute constant $C_{0}$ such that if $|\chi(\sigma+i t)|=1$ with $0 \leq \sigma \leq 1$ and $|t| \geq C_{0}$, then $\sigma=\frac{1}{2}$.

Remark. One can show that the minimal such $C_{0}$ is less than 6.3 .

Proof. Taking the logarithmic derivative of (6.1) by means of Cauchy's integral formula, we find that

$$
\operatorname{Re} \frac{\chi^{\prime}}{\chi}(s)=-\log \frac{\tau}{2 \pi}+O\left(\frac{1}{\tau}\right) .
$$

Since $\left|\chi\left(\frac{1}{2}+i t\right)\right|=1$, we see that if $\sigma_{1}>\frac{1}{2}$, then

$$
\begin{aligned}
\log \left|\chi\left(\sigma_{1}+i t\right)\right| & =\int_{\frac{1}{2}}^{\sigma_{1}} \operatorname{Re} \frac{\chi^{\prime}}{\chi}(s) d \sigma \\
& =\left(\frac{1}{2}-\sigma_{1}\right) \log \frac{\tau}{2 \pi}+O\left(\frac{\sigma_{1}-\frac{1}{2}}{\tau}\right) .
\end{aligned}
$$

This is negative for all $t$ sufficiently large (independent of $\sigma_{1}$ ), so the result follows. The proof is similar for $\sigma_{1}<\frac{1}{2}$.

From now on $C_{0}$ will denote the constant in Lemma 6.1,

We now prove

Theorem 6.2 (The Riemann Hypothesis for $\zeta_{X}(s)$ ). Let $\rho_{X}=\beta_{X}+i \gamma_{X}$ denote any zero of $\zeta_{X}(s)$ with $0 \leq \beta_{X} \leq 1$ and $\gamma_{X} \geq C_{0}$, the constant in Lemma 6.1, Then $\beta_{X}=\frac{1}{2}$.

Proof. Since $\zeta_{X}(s)=P_{X}(s)+\chi(s) P_{X}(\bar{s})$ and $P_{X}(s)$ never vanishes, the zeros of $\zeta_{X}(s)$ can only occur at points where $\left|P_{X}(s)\right|=\left|\chi(s) P_{X}(\bar{s})\right|$, that is, where $|\chi(s)|=$ 1. The result now follows from Lemma 6.1 


\section{The Number of Zeros of $\zeta_{X}(s)$}

In this section we estimate the number of zeros of $\zeta_{X}(s)$ up to height $t$ on the critical line, showing among other things that it has at least as many zeros (essentially) as $\zeta(s)$ does, namely

$$
N(t)=\frac{t}{2 \pi} \log \frac{t}{2 \pi}-\frac{t}{2 \pi}+\frac{7}{8}+S(t)+O\left(\frac{1}{\tau}\right) .
$$

An exact expression for $N(t)$ is

$$
N(t)=-\frac{1}{2 \pi} \arg \chi\left(\frac{1}{2}+i t\right)+S(t)+1,
$$

and we shall use this later. Here the argument of $\chi$ is determined by starting with the value 0 at $s=2$ and letting it vary continuously, first along the segment from 2 to $2+i t$ and then horizontally from $2+i t$ to $\frac{1}{2}+i t$.

To investigate the zeros of $\zeta_{X}(s)$ we write

$$
\zeta_{X}(s)=P_{X}(s)\left(1+\chi(s) \frac{P_{X}(\bar{s})}{P_{X}(s)}\right) .
$$

Since $P_{X}(s)$ is never zero, $\zeta_{X}(s)$ vanishes if and only if $\chi(s) P_{X}(\bar{s}) / P_{X}(s)=-1$. Now $\left|P_{X}(\bar{s}) / P_{X}(s)\right|=1$, so this is equivalent to $|\chi(s)|=1$ and $\arg \left(\chi(s) P_{X}(\bar{s}) / P_{X}(s)\right)$ $\equiv \pi(\bmod 2 \pi)$. By Lemma 6.1] if $|\chi(s)|=1$ in the half-strip $0 \leq \sigma \leq 1, t \geq C_{0}$, then $\sigma=\frac{1}{2}$. Conversely, we know that $\left|\chi\left(\frac{1}{2}+i t\right)\right|=1$ for all $t$. Thus, $\zeta_{X}(s)=0$ in $0 \leq \sigma \leq 1, t \geq C_{0}$ if and only if $\arg \left(\chi\left(\frac{1}{2}+i t\right) P_{X}\left(\frac{1}{2}-i t\right) / P_{X}\left(\frac{1}{2}+i t\right)\right) \equiv \pi$ $(\bmod 2 \pi)$. Defining

$$
F_{X}(t)=-\arg \chi\left(\frac{1}{2}+i t\right)+2 \arg P_{X}\left(\frac{1}{2}+i t\right),
$$

we see that when $t \geq C_{0}, \zeta_{X}\left(\frac{1}{2}+i t\right)=0$ if and only if

$$
F_{X}(t) \equiv \pi(\bmod 2 \pi) \text {. }
$$

This will be the basis for much of our further work.

Before turning to our first estimate, we point out that, as with $\arg \chi\left(\frac{1}{2}+i t\right)$, $\arg P_{X}\left(\frac{1}{2}+i t\right)$ is defined by continuous variation along the segments $[2,2+i t]$ and $\left[2+i t, \frac{1}{2}+i t\right]$, starting with the value 0 at $s=2$. Also note from (7.4) that $F_{X}(t)$ is infinitely differentiable for $t>0$.

We now prove

Theorem 7.1. Let $N_{X}(t)$ denote the number of zeros $\rho=\frac{1}{2}+i \gamma_{X}$ of $\zeta_{X}(s)$ with $0 \leq \gamma_{X} \leq t$. Then

$$
N_{X}(t) \geq \frac{t}{2 \pi} \log \frac{t}{2 \pi}-\frac{t}{2 \pi}-\frac{1}{\pi} \sum_{n \leq X^{2}} \frac{\Lambda_{X}(n) \sin (t \log n)}{n^{\frac{1}{2}} \log n}+O_{X}(1) .
$$

Proof. There are at most finitely many zeros (the number may depend on $X$ ) with ordinates between 0 and $C_{0}$ (the constant in Lemma 6.1). We may therefore assume that $t \geq C_{0}$. Now, by (6.1)

and by (3.4)

$$
\arg \chi\left(\frac{1}{2}+\mathrm{i} t\right)=-t \log \frac{t}{2 \pi}+t+\frac{1}{4} \pi+O\left(\frac{1}{\tau}\right)
$$

$$
\arg P_{X}\left(\frac{1}{2}+i t\right)=\operatorname{Im} \sum_{n \leq X^{2}} \frac{\Lambda_{X}(n)}{n^{\frac{1}{2}+i t} \log n}=-\sum_{n \leq X^{2}} \frac{\Lambda_{X}(n) \sin (t \log n)}{n^{\frac{1}{2}} \log n} .
$$


Thus, we may express $F_{X}(t)$ in (7.4) as

$$
F_{X}(t)=t \log \frac{t}{2 \pi}-t-\frac{1}{4} \pi-2 \sum_{n \leq X^{2}} \frac{\Lambda_{X}(n) \sin (t \log n)}{n^{\frac{1}{2}} \log n}+O\left(\frac{1}{\tau}\right) .
$$

Recall that $\zeta_{X}\left(\frac{1}{2}+\mathrm{i} t\right)=0$ when $t \geq C_{0}$ if and only if $F_{X}(t) \equiv \pi(\bmod 2 \pi)$. Since $F_{X}(t)$ is continuous, this happens at least

$$
\frac{t}{2 \pi} \log \frac{t}{2 \pi}-\frac{t}{2 \pi}-\frac{1}{\pi} \sum_{n \leq X^{2}} \frac{\Lambda_{X}(n) \sin (t \log n)}{n^{\frac{1}{2}} \log n}+O_{X}(1)
$$

times as on $\left[C_{0}, t\right]$. This gives the result.

The sum over prime powers in (7.5) obviously plays an important role in producing zeros of $\zeta_{X}(s)$. This sum is just $-\arg P_{X}\left(\frac{1}{2}+i t\right)$, but it will be convenient to give it a simpler name. Thus, from now on we write

$$
f_{X}(t)=-\arg P_{X}\left(\frac{1}{2}+i t\right)=\sum_{n \leq X^{2}} \frac{\Lambda_{X}(n) \sin (t \log n)}{n^{\frac{1}{2}} \log n} .
$$

As is well known (see Selberg [24] or Titchmarsh 25]), $-(1 / \pi) f_{X}(t)$ is a good approximation in mean-square to $S(t)=(1 / \pi) \arg \zeta\left(\frac{1}{2}+i t\right)$ if $X$ is a small power of $t$ and if the Riemann Hypothesis holds. A closer analogue of $S(t)$ in our context is

$$
S_{X}(t)=\frac{1}{\pi} \arg \zeta_{X}\left(\frac{1}{2}+i t\right)
$$

From (7.3) we see that

$$
\begin{aligned}
S_{X}(t) & =-\frac{1}{\pi} \sum_{n \leq X^{2}} \frac{\Lambda_{X}(n) \sin (t \log n)}{n^{\frac{1}{2}} \log n}-\frac{1}{\pi} \arg \left(1+\chi\left(\frac{1}{2}+i t\right) \frac{P_{X}\left(\frac{1}{2}-i t\right)}{P_{X}\left(\frac{1}{2}+i t\right)}\right) \\
& =-\frac{1}{\pi} f_{X}(t)-\frac{1}{\pi} \arg \left(1+e^{-i F_{X}(t)}\right) .
\end{aligned}
$$

The second term on the right, which contains the jump discontinuities of $S_{X}(t)$ as $t$ passes through zeros of $\zeta_{X}\left(\frac{1}{2}+i t\right)$, has modulus $\leq \frac{1}{2}$. (Note that our convention is that the argument is $\pi / 2$ when $1+e^{-i F_{X}(t)}$ vanishes.) Thus, $S_{X}(t)$ and $-(1 / \pi) f_{X}(t)$ differ by at most $O(1)$.

The next theorem shows that when $X$ is not too small, $f_{X}(t)$ and $S_{X}(t)$ have the same bound as $S(t)$, namely $\Phi(t)$.

Theorem 7.2. Assume the Riemann Hypothesis and that $2 \leq X \leq t^{2}$. Then

$$
f_{X}(t)=\sum_{n \leq X^{2}} \frac{\Lambda_{X}(n) \sin (t \log n)}{n^{\frac{1}{2}} \log n} \ll \Phi(t)+\frac{\log \tau}{\log X} .
$$

In particular, $f_{X}(t) \ll \Phi(t)$ when $\exp (\log \tau / \Phi(t)) \leq X \leq t^{2}$. The same bounds hold for $S_{X}(t)$.

Proof. Since $S_{X}(t)+(1 / \pi) f_{X}(t) \ll 1$, it suffices to prove the result for $f_{X}(t)$. By (3.18) and (3.19) we have

$$
S(t)=-\frac{1}{\pi} \sum_{n \leq X^{2}} \frac{\Lambda_{X}(n) \sin (t \log n)}{n^{\frac{1}{2}} \log n}+O(\Phi(t))+O\left(\frac{\log \tau}{\log X}\right) .
$$

Since $S(t) \ll \Phi(t)$, the result follows. 
From Theorem 7.2 and Theorem 7.1 we immediately obtain

Theorem 7.3. Assume the Riemann Hypothesis is true. Then for $2 \leq X \leq t^{2}$,

$$
N_{X}(t) \geq \frac{t}{2 \pi} \log \frac{t}{2 \pi}-\frac{t}{2 \pi}+O(\log \tau) .
$$

Moreover, if $\exp (c \log \tau / \Phi(t)) \leq X \leq t^{2}$, where $c$ is any positive constant, then

$$
N_{X}(t) \geq \frac{t}{2 \pi} \log \frac{t}{2 \pi}-\frac{t}{2 \pi}+O(\Phi(t))
$$

To obtain an upper bound for $N_{X}(t)$ of the same order we require the following theorem.

Theorem 7.4. Assume the Riemann Hypothesis and that $2 \leq X \leq t^{2}$. Then

$$
f_{X}^{\prime}(t)=\sum_{n \leq X^{2}} \frac{\Lambda_{X}(n) \cos (t \log n)}{n^{\frac{1}{2}}} \ll \Phi(\tau) \log X+\frac{\log \tau}{\log X} .
$$

Proof. Taking the real part of (3.8) with $\sigma=\frac{1}{2}$, we obtain

$$
\begin{aligned}
\operatorname{Re} \frac{\zeta^{\prime}}{\zeta}\left(\frac{1}{2}+i t\right)= & \sum_{\gamma} \frac{\cos ((\gamma-t) \log X)-\cos (2(\gamma-t) \log X)}{(\gamma-t)^{2} \log X} \\
& -\sum_{n \leq X^{2}} \frac{\Lambda_{X}(n) \cos (t \log n)}{\sqrt{n}}+O\left(\frac{1}{\log X}\right) .
\end{aligned}
$$

Similarly, from

$$
\frac{\zeta^{\prime}}{\zeta}(s)=\sum_{\rho}\left(\frac{1}{s-\rho}+\frac{1}{\rho}\right)-\frac{1}{2} \log \frac{\tau}{2 \pi}+O(1)
$$

we see that

$$
\operatorname{Re} \frac{\zeta^{\prime}}{\zeta}(s)=-\frac{1}{2} \log \frac{\tau}{2 \pi}+O(1)
$$

We substitute this into the left-hand side of (17.7) and rearrange the equation to see that

$$
\begin{aligned}
\sum_{n \leq X^{2}} \frac{\Lambda_{X}(n) \cos (t \log n)}{\sqrt{n}}= & \frac{1}{2} \log \frac{\tau}{2 \pi}+\sum_{\gamma} \frac{\cos ((\gamma-t) \log X)-\cos (2(\gamma-t) \log X)}{(\gamma-t)^{2} \log X} \\
& +O(1) .
\end{aligned}
$$

In the sum over zeros, the terms with $|t-\gamma| \geq 1$ contribute $\ll \log \tau / \log X$. Thus, writing $C(v)=\cos (v \log X)-\cos (2 v \log X)$, we have

$$
\sum_{n \leq X^{2}} \frac{\Lambda_{X}(n) \cos (t \log n)}{\sqrt{n}}=\frac{1}{2} \log \frac{\tau}{2 \pi}+\frac{1}{\log X} \sum_{|\gamma-t| \leq 1} \frac{C(\gamma-t)}{(\gamma-t)^{2}}+O\left(\frac{\log \tau}{\log X}\right)
$$

To estimate the sum on the last line, first note that

$$
C(v)= \begin{cases}\frac{3}{2} v^{2} \log ^{2} X+O\left(|v|^{4} \log ^{4} X\right) & \text { if } \quad|v| \leq 1 / \log X \\ O(1) & \text { if } \quad|v|>1 / \log X\end{cases}
$$

and that

$$
C^{\prime}(v)= \begin{cases}3 v \log ^{2} X+O\left(|v|^{3} \log ^{4} X\right) & \text { if } \quad|v| \leq 1 / \log X \\ O(\log X) & \text { if } \quad|v|>1 / \log X\end{cases}
$$


In particular, it follows that

$$
\frac{d}{d v}\left(\frac{C(v)}{v^{2}}\right)=\frac{v^{2} C^{\prime}(v)-2 v C(v)}{v^{4}} \ll\left\{\begin{array}{lll}
|v| \log ^{4} X & \text { if } & |v| \leq 1 / \log X, \\
v^{-2} \log X & \text { if } & |v|>1 / \log X .
\end{array}\right.
$$

Now, by (7.1)

$$
\begin{aligned}
\sum_{|\gamma-t| \leq 1} \frac{C(\gamma-t)}{(\gamma-t)^{2}} & =\int_{|u-t| \leq 1} \frac{C(u-t)}{(u-t)^{2}} d N(u) \\
& =\frac{1}{2 \pi} \int_{|v| \leq 1} \frac{C(v)}{v^{2}} \log \left(\frac{t+v}{2 \pi}\right) d v+\int_{|v| \leq 1} \frac{C(v)}{v^{2}} d S(t+v) .
\end{aligned}
$$

Using (17.10), we see that the integral with respect to $d S$ is

$$
\begin{aligned}
S(t+v) & \left.\frac{C(v)}{v^{2}}\right|_{-1} ^{1}-\int_{|v| \leq 1} S(t+v)\left(\frac{C(v)}{v^{2}}\right)^{\prime} d v \\
& \ll \Phi(\tau)+\Phi(\tau)\left(\int_{|v| \leq 1 / \log X}|v| \log ^{4} X d v+\int_{1 / \log X<|v| \leq 1} \frac{\log X}{v^{2}} d v\right) \\
& \ll \Phi(\tau) \log ^{2} X .
\end{aligned}
$$

The other integral is

$$
\frac{1}{2 \pi} \int_{|v| \leq 1}\left(\log \frac{t}{2 \pi}+O\left(\frac{|v|}{t}\right)\right) \frac{C(v)}{v^{2}} d v .
$$

The $O$-term contributes

$$
\ll \frac{1}{\tau}\left(\log ^{2} X \int_{|v| \leq 1 / \log X}|v| d v+\int_{1 / \log X<|v| \leq 1}|v|^{-1} d v\right) \ll \frac{1}{\tau} \log \log X
$$

by (7.9). Thus, combining these results, we find that

$$
\sum_{|\gamma-t| \leq 1} \frac{C(\gamma-t)}{(\gamma-t)^{2}}=\frac{1}{2 \pi} \log \frac{t}{2 \pi} \int_{|v| \leq 1} \frac{C(v)}{v^{2}} d v+O\left(\Phi(\tau) \log ^{2} X\right) .
$$

To calculate the integral we write

$$
\int_{|v| \leq 1} \frac{C(v)}{v^{2}} d v=\int_{-\infty}^{\infty} \frac{C(v)}{v^{2}} d v-\int_{|v|>1} \frac{C(v)}{v^{2}} d v .
$$

By (7.9) the second integral is $O(1)$. By the calculus of residues and the definition of $C(v)$, the first equals

$$
\begin{aligned}
\int_{-\infty}^{\infty} \frac{\cos (v \log X)-\cos (2 v \log X)}{v^{2}} d v & =\operatorname{Re} \int_{-\infty}^{\infty} \frac{e^{i v \log X}-e^{2 i v \log X}}{v^{2}} d v \\
& =\operatorname{Re} 2 \pi i\left(-\frac{1}{2} \operatorname{Res}_{v=0} \frac{e^{i v \log X}-e^{2 i v \log X}}{v^{2}}\right) \\
& =-\operatorname{Re} \pi i(-i \log X)=-\pi \log X
\end{aligned}
$$

Thus,

$$
\int_{|v| \leq 1} \frac{C(v)}{v^{2}} d v=-\pi \log X+O(1)
$$


Using this in (7.11), we obtain

$$
\sum_{|\gamma-t| \leq 1} \frac{C(\gamma-t)}{(\gamma-t)^{2}}=-\frac{1}{2} \log \frac{t}{2 \pi} \log X+O\left(\Phi(\tau) \log ^{2} X\right)+O(\log \tau) .
$$

It therefore follows from (7.8) that

$$
\sum_{n \leq X^{2}} \frac{\Lambda_{X}(n) \cos (t \log n)}{\sqrt{n}} \ll \Phi(\tau) \log X+\frac{\log \tau}{\log X} .
$$

This completes the proof of the theorem.

The zeros of $\zeta_{X}(\sigma+i t)$ with $t \geq C_{0}$ arise as the solutions of

$$
F_{X}(t) \equiv \pi(\bmod 2 \pi)
$$

and their number in $\left[C_{0}, t\right]$ is at least $(1 / 2 \pi) F_{X}(t)+O_{X}(1)$ because this is the minimum number of times the curve $y=F_{X}(t)$ crosses the horizontal lines $y=$ $\pi, 3 \pi, 5 \pi, \ldots$. However, there could be "extra" solutions if $F_{X}(t)$ is not monotone increasing. Now

$$
F_{X}^{\prime}(t)=\log \frac{t}{2 \pi}-2 \sum_{n \leq X^{2}} \frac{\Lambda_{X}(n) \cos (t \log n)}{n^{\frac{1}{2}}}+O\left(\frac{1}{\tau}\right) .
$$

By Theorem 7.4 there exists a positive constant $C_{3}$, say, such that if $X \leq$ $\exp \left(C_{3} \log \tau / \Phi(t)\right)$ and $t$ is large enough, then

$$
\sum_{n \leq X^{2}} \frac{\Lambda_{X}(n) \cos (t \log n)}{n^{\frac{1}{2}}}<\frac{1}{2} \log \frac{t}{2 \pi} .
$$

This means $F_{X}^{\prime}(t)$ is positive, so $F_{X}(t) \equiv \pi(\bmod 2 \pi)$ has no extra solutions. We have therefore proved

Theorem 7.5. Assume the Riemann Hypothesis. There is a constant $C_{3}>0$ such that if $X<\exp \left(C_{3} \log t / \Phi(t)\right)$, then

$$
N_{X}(t)=\frac{t}{2 \pi} \log \frac{t}{2 \pi}-\frac{t}{2 \pi}-\frac{1}{\pi} \sum_{n \leq X^{2}} \frac{\Lambda_{X}(n) \sin (t \log n)}{n^{\frac{1}{2}} \log n}+O_{X}(1) .
$$

Less precisely,

$$
N_{X}(t)=\frac{t}{2 \pi} \log \frac{t}{2 \pi}-\frac{t}{2 \pi}+O_{X}(\Phi(\tau)) .
$$

It would be useful to know whether (7.14) (perhaps with a larger $O$-term) also holds when $X$ is a small fixed power of $t$. If that is the case, classical results about the statistics of the zeros of $\zeta(s)$ whose proofs depend on approximating $S(t)$ by the trigonometric polynomial $-(1 / \pi) f_{X}(t)$ would hold for the zeros of $\zeta_{X}(s)$ as well. What we can show for larger $X$ is the following unconditional result.

Theorem 7.6. There exists a positive constant $C_{4}$ such that if $X \leq t^{C_{4}}$, then

$$
N_{X}(t) \ll t \log t
$$

Moreover, if $X \leq t^{o(1)}$, then

$$
N_{X}(t)=(1+o(1)) \frac{t}{2 \pi} \log \frac{t}{2 \pi} .
$$


Proof. There are two ways that solutions to $F_{X}(t) \equiv \pi(\bmod 2 \pi)$ may arise, and we shall refer to the zeros of $\zeta_{X}(s)$ corresponding to these two ways as zeros of the "first" and "second" kind.

The first way is by $F_{X}(t)$ increasing or decreasing from one odd multiple of $\pi$, say $(2 k+1) \pi$, to the next larger or smaller odd multiple of $\pi$, without first crossing $(2 k+1) \pi$ again. A moment's reflection reveals that the total number of distinct zeros in $\left[C_{0}, t\right]$ arising this way is big- $O$ of the total variation of $F_{X}(t)$, namely,

$$
\frac{1}{2 \pi} \int_{C_{0}}^{t}\left|F_{X}^{\prime}(u)\right| d u
$$

By (7.12) and the triangle and Cauchy-Schwarz inequalities, this is

$$
\begin{aligned}
& \leq \frac{1}{2 \pi} \int_{C_{0}}^{t}\left|\log \frac{u}{2 \pi}-2 \sum_{n \leq X^{2}} \frac{\Lambda_{X}(n) \cos (u \log n)}{\sqrt{n}}\right| d u+O(1) \\
& \leq \frac{t}{2 \pi} \log \frac{t}{2 \pi}-\frac{t}{2 \pi}+\frac{1}{\pi} t^{1 / 2}\left(\int_{C_{0}}^{t}\left|\sum_{n \leq X^{2}} \frac{\Lambda_{X}(n) \cos (u \log n)}{\sqrt{n}}\right|^{2} d u\right)^{1 / 2} .
\end{aligned}
$$

By a standard mean value theorem for Dirichlet polynomials it is easy to show that if $X \ll t^{1 / 2}$, the integral is $\ll t \log ^{2} X$. Thus, writing $N_{I}(t)$ for the number of distinct zeros that occur in this way, we have

$$
N_{I}(t) \leq \frac{t}{2 \pi} \log \frac{t}{2 \pi}-\frac{t}{2 \pi}+O(t \log X)
$$

We will see how to take multiplicities into account below.

The second way solutions to $F_{X}(t) \equiv \pi(\bmod 2 \pi)$ can occur is by $F_{X}(t)$ increasing or decreasing from a solution $(2 k+1) \pi$ and returning to this value before reaching the next larger or smaller odd multiple of $\pi$. Each time this happens, there must be at least one point in between where $F_{X}^{\prime}(t)$ vanishes. Thus, writing $N_{I I}(t)$ for the number of distinct zeros of $\zeta_{X}(s)$ arising this way, we see that $N_{I I}(t)$ is at most big- $O$ of the number of times $F_{X}^{\prime}(t)$ vanishes on $\left[C_{0}, t\right]$. To estimate this number we define functions

$$
g_{X}(s)=-\frac{\chi^{\prime}}{\chi}(s)-2 \sum_{n \leq X^{2}} \frac{\Lambda_{X}(n)}{n^{s}}
$$

and

$$
G_{X}(s)=\frac{1}{2}\left(g_{X}(s)+g_{X}(1-s)\right) .
$$

By (7.4), $F_{X}^{\prime}(t)=G_{X}\left(\frac{1}{2}+i t\right)$, so the zeros of $F_{X}^{\prime}(t)$ on $\left[C_{0}, t\right]$ are the zeros of $G_{X}(s)$ on $\left[\frac{1}{2}+i C_{0}, \frac{1}{2}+i t\right]$. We bound this number by bounding the number of zeros on each of the segments $\left[\frac{1}{2}+i t, \frac{1}{2}+2 i t\right],\left[\frac{1}{2}+i \frac{t}{2}, \frac{1}{2}+i t\right], \ldots$, and adding. The number of zeros on any one of these is at most the number of zeros of $G_{X}(s)$ in a disk containing the segment. By a familiar result from complex analysis, if $\mathcal{D}$ is a closed disk of radius $R$ centered at $z_{0}, f(z)$ is analytic on $\mathcal{D}$ with maximum modulus $M$, and $f\left(z_{0}\right) \neq 0$, then there is an absolute constant $c$ such that $f$ has $\leq c \log \left(M /\left|f\left(z_{0}\right)\right|\right)$ zeros in the disc of radius $\frac{2}{3} R$ centered at $z_{0}$. To apply this to the segment $\left[\frac{1}{2}+i t, \frac{1}{2}+2 i t\right]$, say, we need a disc containing it, the maximum of $\left|G_{X}(s)\right|$ on this disk, and a lower bound for $\left|G_{X}\right|$ at the center of the disk. We handle the last problem first by selecting as center a point at which we know $\left|G_{X}(s)\right|$ cannot 
be too small. The upper bound for $N_{I I}(t)$ will follow by repeating this process for each of the segments and adding the resulting estimates.

To show that one can find a satisfactory center, fix a $\delta$ with $0<\delta<\frac{1}{2}$ and set

$$
\mathcal{E}(t)=\left\{u \in[t, 2 t]:\left(\frac{1}{2}+\delta\right) \log \frac{t}{2 \pi} \geq\left|f_{X}^{\prime}(u)\right| \geq\left(\frac{1}{2}-\delta\right) \log \frac{t}{2 \pi}\right\} .
$$

Then $\left|F_{X}^{\prime}(u)\right|=\left|G_{X}\left(\frac{1}{2}+i u\right)\right| \leq 2 \delta \log (t / 2 \pi)+O(1 / t)$ for all $u \in \mathcal{E}(t)$. Now recall that

$$
\int_{t}^{2 t}\left|f_{X}^{\prime}(u)\right|^{2} d u=\int_{t}^{2 t}\left|\sum_{n \leq X^{2}} \frac{\Lambda_{X}(n) \cos (u \log n)}{\sqrt{n}}\right|^{2} d u \ll t \log ^{2} X .
$$

Thus, the measure of $\mathcal{E}(t)$ is

$$
\begin{aligned}
|\mathcal{E}(t)|=\int_{\mathcal{E}(t)} 1 d t & \leq \int_{\mathcal{E}(t)}\left(\frac{\left|f_{X}^{\prime}(u)\right|}{\left(\frac{1}{2}-\delta\right) \log \frac{t}{2 \pi}}\right)^{2} d t \\
& \ll \frac{t \log ^{2} X}{\log ^{2} t} .
\end{aligned}
$$

It follows that there exists a constant $C_{4}>0$ such that if $X \leq t^{C_{4}}$, then $|\mathcal{E}(t)|<\frac{1}{6} t$. Since the segment $\left[\frac{1}{2}+i\left(\frac{3}{2}-\frac{1}{12}\right) t, \frac{1}{2}+i\left(\frac{3}{2}+\frac{1}{12}\right) t\right]$ has greater length than the set $\mathcal{E}(t)$, it contains a point $\frac{1}{2}+i t_{0}$ with $t_{0}$ not in $\mathcal{E}(t)$ and therefore with $\left|G_{X}\left(\frac{1}{2}+i t_{0}\right)\right|>$ $2 \delta \log (t / 2 \pi)$.

We now let $\mathcal{D}_{0}(t)$ be the closed disc of radius $t$ centered at $\frac{1}{2}+i t_{0}$, and let $M$ denote the maximum of $\left|G_{X}(s)\right|$ on $\mathcal{D}_{0}(t)$. Clearly on $\mathcal{D}_{0}(t)$ we have

$$
G_{X}(\sigma+i t) \ll \log t+\sum_{n \leq X^{2}} \Lambda_{X}(n) n^{t-1 / 2} \ll X^{t+1 / 2} .
$$

Hence, by the theorem alluded to above, $G_{X}(s)$ has $\ll \log \left(X^{t+1 / 2} / \delta \log t\right) \ll t \log X$ zeros inside the smaller disc $\mathcal{D}_{0}\left(\frac{2}{3} t\right)$ of radius $\frac{2}{3} t$, which covers $\left[\frac{1}{2}+i t, \frac{1}{2}+2 i t\right]$. Adding estimates for the different intervals, we arrive at $\ll t \log X$ distinct zeros of $F_{X}^{\prime}(t)$. The same bound therefore holds for the number of distinct zeros of the second kind.

Combining the two ways the solutions of $F_{X}(t) \equiv \pi(\bmod 2 \pi)$ or zeros of $\zeta_{X}(s)$ arise, we find that for $X \leq t^{C_{4}}$ there are

$$
N_{I}(t)+N_{I I}(t) \leq(t / 2 \pi) \log (t / 2 \pi)-(t / 2 \pi)+O(t \log X)
$$

distinct zeros.

Now, a zero $\frac{1}{2}+i \gamma_{X}$ of $\zeta_{X}\left(\frac{1}{2}+i t\right)$ has multiplicity $m$ if and only if the first $m-1$ derivatives of $\zeta_{X}\left(\frac{1}{2}+i t\right)$ with respect to $t$ vanish at $\gamma_{X}$, but the $m t h$ does not. It is easy to check that this is equivalent to $F_{X}\left(\gamma_{X}\right) \equiv \pi(\bmod 2 \pi), F_{X}^{\prime}\left(\gamma_{X}\right)=$ $\ldots=F_{X}^{(m-1)}\left(\gamma_{X}\right)=0$, and $F_{X}^{(m)}\left(\gamma_{X}\right) \neq 0$. Also note that our estimate for the number of zeros of the analytic function $G_{X}(s)$ counts them according to their multiplicities, and that $F_{X}^{\prime}(t)=G_{X}\left(\frac{1}{2}+i t\right), F_{X}^{(2)}(t)=i G_{X}^{(1)}\left(\frac{1}{2}+i t\right), \ldots, F_{X}^{(m)}(t)=$ $i^{m-1} G_{X}^{(m-1)}\left(\frac{1}{2}+i t\right)$.

Suppose then that $\frac{1}{2}+i \gamma_{X}$ is a zero of $\zeta_{X}(s)$ of the first kind and multiplicity $m$. Then it is counted once in $N_{I}(t)$. Also, since the first $m-1$ derivatives of $F_{X}(t)$ vanish at $\gamma_{X}$, so does $G_{X}\left(\frac{1}{2}+i t\right)$ and its first $m-2$ derivatives. Thus, $\frac{1}{2}+i \gamma_{X}$ is counted another $m-1$ times in $N_{I I}(t)$, and therefore with the correct multiplicity in $N_{I}(t)+N_{I I}(t)$. 
Next suppose that $\frac{1}{2}+i \gamma_{X}$ is a zero of multiplicity $m$ of the second kind. Then it is counted at least once in $N_{I I}(t)$ because $F_{X}^{\prime}(t)=G_{X}\left(\frac{1}{2}+i t\right)$ vanishes at a nearby point. Also, at $\gamma_{X}$ itself we have $F_{X}^{\prime}\left(\gamma_{X}\right)=\cdots=F_{X}^{m-1}\left(\gamma_{X}\right)=0$, and $F_{X}^{m}\left(\gamma_{X}\right) \neq 0$. This means that $G_{X}(s)$ and its first $m-2$ derivatives are zero at $\frac{1}{2}+i \gamma_{X}$, so this point is counted $m-1$ times by $N_{I I}(t)$. Thus, zeros of the second kind with multiplicity $m$ are counted with weight at least $m$ in $N_{I I}(t)$.

We now see that

$$
N_{X}(t) \leq \frac{t}{2 \pi} \log \frac{t}{2 \pi}-\frac{t}{2 \pi}+O(t \log X) .
$$

Both assertions of the theorem now follow from this and the lower bound in (7.6).

\section{The number of Simple zeros of $\zeta_{X}(s)$}

We saw in the last section that a zero $\frac{1}{2}+i \gamma_{X}$ of $\zeta_{X}(s)$ is simple if and only if $F_{X}\left(\gamma_{X}\right) \equiv \pi(\bmod 2 \pi)$ and $F_{X}^{\prime}\left(\gamma_{X}\right) \neq 0$. Let $N_{X}^{(1)}(t)$ denote the number of such zeros up to height $t$. From (7.12) and (7.13) we see that $F_{X}^{\prime}\left(\gamma_{X}\right)>0$ if $X$ is not too large, and therefore that $\frac{1}{2}+i \gamma_{X}$ is a simple zero of $\zeta_{X}(s)$. Combining this with Theorem 7.5, we obtain

Theorem 8.1. Assume the Riemann Hypothesis. There exists a constant $C_{3}>0$ such that if $X<\exp \left(C_{3} \log t / \Phi(t)\right)$, then all the zeros of $\zeta_{X}\left(\frac{1}{2}+i t\right)$ with $t \geq C_{0}$ are simple and

$$
N_{X}^{(1)}(t)=N_{X}(t)=\frac{t}{2 \pi} \log \frac{t}{2 \pi}-\frac{t}{2 \pi}-\frac{1}{\pi} \sum_{n \leq X^{2}} \frac{\Lambda_{X}(n) \sin (t \log n)}{n^{1 / 2} \log n}+O_{X}(1) .
$$

As in our results for $N_{X}(t)$, the condition on $X$ is almost certainly too restrictive. The following unconditional but less precise result is valid for larger $X$.

Theorem 8.2. Let $\epsilon>0$ and $X \leq \exp \left(o\left(\log ^{1-\epsilon} t\right)\right)$. Then as $t \rightarrow \infty$, the number of simple zeros up to height $t$ is

$$
N_{X}^{(1)}(t)=(1+o(1)) \frac{t}{2 \pi} \log \frac{t}{2 \pi} .
$$

Proof. Let $N$ be the number of zeros of $\zeta_{X}\left(\frac{1}{2}+i u\right)$ in $[t, 2 t]$ and $N^{*}$ the number of these that are multiple. By Theorem [7.6. there is a constant $C_{4}$ such that if $X \leq t^{C_{4}}$, then $N \ll t \log t$. We may therefore split the $N^{*}$ multiple zeros into $K \ll \log t$ sets $\mathcal{S}_{1}, \mathcal{S}_{2}, \ldots, \mathcal{S}_{K}$, in each of which the points are at least 1 apart. Let $\mathcal{S}$ be one of these sets and let $\gamma_{1}, \gamma_{2}, \ldots, \gamma_{R}$ be its points. Then these must all satisfy

$$
0=F_{X}^{\prime}\left(\gamma_{r}\right)=\log \frac{\gamma_{r}}{2 \pi}-2 \sum_{n \leq X^{2}} \frac{\Lambda_{X}(n) \cos \left(\gamma_{r} \log n\right)}{n^{1 / 2}}+O\left(\frac{1}{\gamma_{r}}\right) .
$$

Writing

$$
\sum_{n \leq X^{2 k}} \frac{A_{X}(n)}{n^{1 / 2+i u}}=\left(\sum_{n \leq X^{2}} \frac{\Lambda_{X}(n)}{n^{1 / 2+i u}}\right)^{k}
$$

we have by a mean value theorem of Davenport (Montgomery [20]) that

$$
\sum_{r=1}^{R}\left|\sum_{n \leq X^{2}} \frac{\Lambda_{X}(n)}{n^{1 / 2+i \gamma_{r}}}\right|^{2 k} \ll\left(t+X^{2 k} \log \left(X^{2 k}\right)\right) \log \left(X^{2 k}\right) \sum_{n \leq X^{2 k}} \frac{\left|A_{X}(n)\right|^{2}}{n} .
$$



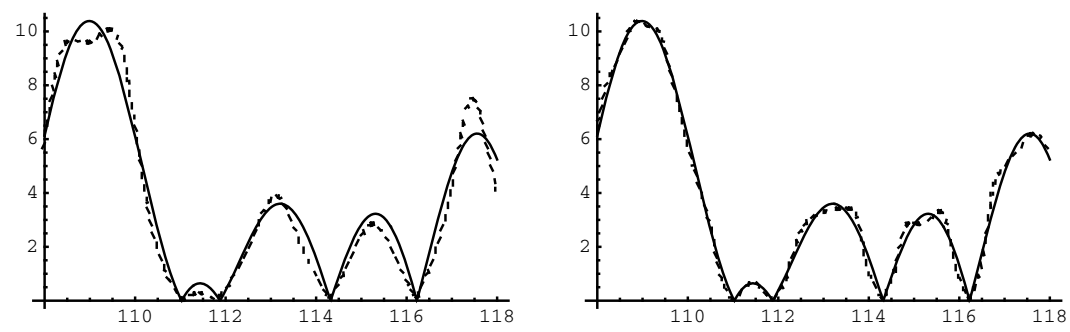

FigURE 1. Graphs of $2\left|\zeta\left(\frac{1}{2}+i t\right)\right|$ (solid) and $\left|\zeta_{X}\left(\frac{1}{2}+i t\right)\right|$ (dotted) near $t=114$ for $X=10$ and $X=300$, respectively.
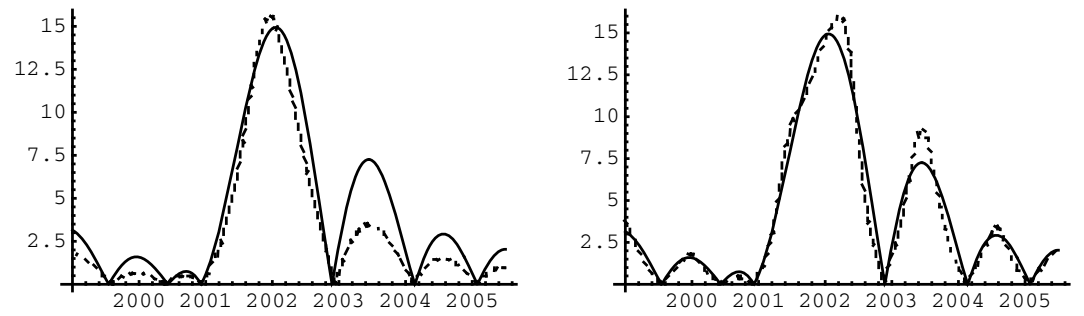

Figure 2. Graphs of $2\left|\zeta\left(\frac{1}{2}+i t\right)\right|$ (solid) and $\left|\zeta_{X}\left(\frac{1}{2}+i t\right)\right|$ (dotted) near $t=2000$ for $X=10$ and $X=300$, respectively.

It is not difficult to show that the sum on the right is $\ll_{2 k} \log ^{2 k} X$ so, if $X \leq t^{1 / 2 k}$, the right-hand side is $\ll_{k} t \log ^{2 k+2} X$. (We also require $X \leq t^{C_{4}}$, so we assume that $k \geq C_{4} / 2$.) On the other hand, by (8) the left-hand side must be $\gg R \log ^{2 k} t$. Therefore $|\mathcal{S}|=R \ll_{k} t \log ^{2 k+2} X / \log ^{2 k} t$. There are $K \ll \log t$ sets $\mathcal{S}_{k}$, so the total possible number of multiple zeros is $N^{*} \ll_{k} t\left(\log ^{2 k+2} X / \log ^{2 k-1} t\right)$. This is $o_{k}(t \log t)$ if $X \leq \exp \left(o\left((\log t)^{1-1 /(k+1)}\right)\right)$. Taking $k$ large enough so that $1 /(k+1)<$ $\epsilon$, we obtain the result.

\section{The Relative sizes of $\zeta_{X}(s)$ And $\zeta(s)$ AND the RElation BETWEEN THEIR ZEROS}

Although we have not proved that $\zeta_{X}(s)$ approximates $\zeta(s)$ pointwise when $\sigma$ is very close to $\frac{1}{2}$, the similarity between the formulae for $N_{X}(t)$ and $N(t)$ suggests there might be a close relationship between the two functions even on the critical line. Indeed, comparing the graphs of $\left|\zeta_{X}\left(\frac{1}{2}+i t\right)\right|$ and $\left|\zeta\left(\frac{1}{2}+i t\right)\right|$ for a wide range of $X$ and $t$ (see Figures 1 and 2), one is struck by two things:

i) the zeros of $\zeta_{X}\left(\frac{1}{2}+i t\right)$ are quite close to those of $\zeta\left(\frac{1}{2}+i t\right)$, even for relatively small $X$, and

ii) as $X$ increases, $\left|\zeta_{X}\left(\frac{1}{2}+i t\right)\right|$ seems to approach $2\left|\zeta\left(\frac{1}{2}+i t\right)\right|$.

An explanation for the second observation is that although $\zeta_{X}(s)$ approximates $\zeta(s)$ to the right of the critical line, so does $P_{X}(s)$. Therefore $\zeta_{X}(s)=P_{X}(s)+$ $\chi(s) P_{X}(\bar{s})$ might be a closer approximation to the function $\mathcal{F}(s)=\zeta(s)+\chi(s) \zeta(\bar{s})$ than to $\zeta(s)$. If this is the case, then on the critical line we have by the functional 
equation that

$$
\begin{aligned}
\zeta_{X}\left(\frac{1}{2}+i t\right) \approx \mathcal{F}\left(\frac{1}{2}+i t\right) & =\zeta\left(\frac{1}{2}+i t\right)+\chi\left(\frac{1}{2}+i t\right) \zeta\left(\frac{1}{2}-i t\right) \\
& =\zeta\left(\frac{1}{2}+i t\right)+\left|\chi\left(\frac{1}{2}+i t\right)\right|^{2} \zeta\left(\frac{1}{2}+i t\right) \\
& =2 \zeta\left(\frac{1}{2}+i t\right) .
\end{aligned}
$$

To establish both observations rigorously we need to introduce a slightly modified version of $\zeta_{X}(s)$. Let

$$
P_{X}^{*}(s)=P_{X}(s) \exp \left(-F_{2}((s-1) \log X)\right)
$$

and define

Note that by (3.24),

$$
\zeta_{X}^{*}(s)=P_{X}^{*}(s)+\chi(s) P_{X}^{*}(\bar{s})
$$

$$
P_{X}^{*}(s)=P_{X}(s) \exp \left(O\left(\frac{X^{2-2 \sigma}}{\tau^{2} \log ^{2} X}\right)\right)
$$

when $\sigma \geq \frac{1}{2}$ and $|s-1| \geq \frac{1}{10}$. The difference between $P_{X}(s)$ and $P_{X}^{*}(s)$, and so also $\zeta_{X}(s)$ and $\zeta_{X}^{*}(s)$, is small when $2 \leq X \leq t^{2}$, as we have been assuming until now. We need to take $X$ much larger, though, in what follows. Similarly, we replace $F_{X}(t)$ by

$$
\begin{aligned}
F_{X}^{*}(t) & =-\arg \chi\left(\frac{1}{2}+i t\right)+2 \arg P_{X}^{*}\left(\frac{1}{2}+i t\right) \\
& =-\arg \chi\left(\frac{1}{2}+i t\right)+2\left(\arg P_{X}\left(\frac{1}{2}+i t\right)-\operatorname{Im} F_{2}\left(\left(-\frac{1}{2}+i t\right) \log X\right)\right) .
\end{aligned}
$$

By (7.4) and (3.24)

$$
F_{X}^{*}(t)=F_{X}(t)+O\left(\frac{X}{\tau^{2} \log X}\right),
$$

so these two functions are also close when $X \leq t^{2}$. The zeros of $\zeta_{X}^{*}\left(\frac{1}{2}+i t\right)$ are the solutions of $F_{X}^{*}(t) \equiv \pi(\bmod 2 \pi)$, and we will show that $\left.i\right)$ and $\left.i i\right)$ above hold provided we use $\zeta_{X}^{*}\left(\frac{1}{2}+i t\right)$ in place of $\zeta_{X}\left(\frac{1}{2}+i t\right)$.

Assume the Riemann Hypothesis is true. Taking the argument of both sides of (3.7) and recalling that $S(t)=(1 / \pi) \arg \zeta\left(\frac{1}{2}+i t\right)$, we see that

$$
\begin{gathered}
\pi S(t)=\arg P_{X}\left(\frac{1}{2}+i t\right)-\operatorname{Im} F_{2}\left(\left(-\frac{1}{2}+i t\right) \log X\right)+\sum_{\gamma} \operatorname{Im} F_{2}(i(t-\gamma) \log X) \\
+O\left(\frac{X^{-\frac{3}{2}}}{\tau^{2} \log X}\right)
\end{gathered}
$$

where $\gamma$ runs through the ordinates of the zeros of $\zeta(s)$. We use this to replace the quantity in parentheses in (9.1) and obtain

$$
F_{X}^{*}(t)=-\arg \chi\left(\frac{1}{2}+i t\right)+2 \pi S(t)-2 \operatorname{Im} \sum_{\gamma} F_{2}(i(t-\gamma) \log X)+O\left(\frac{X^{-\frac{3}{2}}}{\tau^{2} \log X}\right) .
$$

Now, by (7.2)

thus

$$
-\arg \chi\left(\frac{1}{2}+i t\right)+2 \pi S(t)=2 \pi N(t)-2 \pi,
$$

$$
\frac{1}{2 \pi} F_{X}^{*}(t)=N(t)-1-\frac{1}{\pi} \operatorname{Im} \sum_{\gamma} F_{2}(i(t-\gamma) \log X)+O\left(\frac{X^{-\frac{3}{2}}}{\tau^{2} \log X}\right) .
$$


We use this first to show that the zeros of $\zeta_{X}^{*}(s)$ cluster around the zeros of $\zeta(s)$ as $X \rightarrow \infty$. Let $\gamma_{1}$ and $\gamma_{2}$ denote ordinates of distinct consecutive zeros of $\zeta(s)$, and let $0<\Delta \leq \frac{1}{4}\left(\gamma_{1}-\gamma_{2}\right)$. Also, let $\mathcal{I}=\left[\gamma_{1}+\Delta, \gamma_{2}-\Delta\right]$. Then by (3.22) and Lemma 3.3. if $X \geq \exp (1 / \Delta)$ we have

$$
\begin{aligned}
\sum_{\gamma} F_{2}(i(t-\gamma) \log X) & \ll \frac{1}{\log ^{2} X} \sum_{|\gamma-t|>\Delta} \frac{1}{(t-\gamma)^{2}} \\
& \ll \frac{1}{\Delta \log ^{2} X}\left(\log \tau+\frac{\Phi(\tau)}{\Delta}\right)
\end{aligned}
$$

uniformly for $t \in \mathcal{I}$. It now follows from (9.3) that given any $\epsilon>0$, there exists an $X_{0}=X_{0}\left(\gamma_{1}, \Delta, \epsilon\right)$ such that if $X \geq X_{0}$, then

$$
\left\|\frac{F_{X}^{*}(t)}{2 \pi}-N(t)\right\|<\epsilon
$$

uniformly for $t \in \mathcal{I}$. Here $\|x\|$ denotes distance from $x$ to the nearest integer. Since $N(t)$ is an integer when $t \in \mathcal{I}$, this means that if $0<\epsilon<\frac{1}{2}$, then $\frac{1}{2}+i t$ is not a zero of $\zeta_{X}^{*}(s)$. Thus $\mathcal{I}$ is free from zeros of $\zeta_{X}^{*}(s)$ when $X$ is sufficiently large.

Now we show that $\zeta_{X}^{*}\left(\frac{1}{2}+i t\right)$ tends to $2 \zeta\left(\frac{1}{2}+i t\right)$ on $\mathcal{I}$. By (9) and (9.1) we may write

$$
\zeta_{X}^{*}\left(\frac{1}{2}+i t\right)=P_{X}^{*}\left(\frac{1}{2}+i t\right)\left(1+e^{-i F_{X}^{*}(t)}\right)
$$

Also, by Theorem 3.2 and the definitions of $P_{X}^{*}$ and $Z_{X}$, we have

$$
\zeta(s)=P_{X}^{*}(s) \exp \left(\sum_{\gamma} F_{2}(i(t-\gamma) \log X)\right)\left(1+O\left(\frac{X^{-\frac{5}{2}}}{\tau^{2} \log ^{2} X}\right)\right) .
$$

From the first of these and (9.5) we see that for $X$ sufficiently large

$$
\zeta_{X}^{*}\left(\frac{1}{2}+i t\right)=P_{X}^{*}\left(\frac{1}{2}+i t\right)(2+O(\epsilon))
$$

uniformly for $t \in \mathcal{I}$. From the second and (9.4) we see that for $X$ sufficiently large

$$
\zeta\left(\frac{1}{2}+i t\right)=P_{X}^{*}\left(\frac{1}{2}+i t\right)(1+O(\epsilon))
$$

on $\mathcal{I}$. Thus, $\zeta_{X}^{*}\left(\frac{1}{2}+i t\right) \rightarrow 2 \zeta\left(\frac{1}{2}+i t\right)$ as $X \rightarrow \infty$ uniformly for $t \in \mathcal{I}$.

Combining our results we now have

Theorem 9.1. Assume the Riemann Hypothesis. Let $\gamma$ and $\gamma^{\prime}$ denote ordinates of distinct consecutive zeros of the Riemann zeta-function and let $\mathcal{I}$ denote a closed subinterval of $\left(\gamma, \gamma^{\prime}\right)$. Then for all $X$ sufficiently large, $\zeta_{X}^{*}\left(\frac{1}{2}+i t\right)$ has no zeros in $\mathcal{I}$. Moreover, $\zeta_{X}^{*}\left(\frac{1}{2}+i t\right) \rightarrow 2 \zeta\left(\frac{1}{2}+i t\right)$ as $X \rightarrow \infty$ uniformly for $t \in \mathcal{I}$.

E. Bombieri has pointed out to the author that (9.2) is closely related to an explicit formula of Guinand ([11, 12 ), namely,

$$
\begin{aligned}
\pi S(t)= & -\lim _{X \rightarrow \infty}\left(\sum_{n \leq X} \frac{\Lambda(n) \sin (t \log n)}{n^{1 / 2} \log n}-\int_{1}^{X} \frac{\sin (t \log u)}{u^{1 / 2} \log u} d u\right) \\
& -\lim _{X \rightarrow \infty}\left(\frac{\sin (t \log X)}{\log X}\left(\sum_{n \leq X} \frac{\Lambda(n)}{n^{1 / 2}}-2 X^{1 / 2}\right)\right)+\arctan 2 t \\
& -\frac{1}{4} \arctan (\sinh \pi t)-\frac{7}{8} \pi+\frac{1}{2}\left(\arg \Gamma\left(\frac{1}{2}+i t\right)-t \log t+t\right) .
\end{aligned}
$$

There is no sum over zeros here because Guinand is taking a limit. Also, the $\Lambda(n)$ are unweighted. However, this is only a minor difference. 
It is remarkable that the zeros of $\zeta_{X}^{*}(s)$ and $\zeta_{X}(s)$ are close to those of $\zeta(s)$ even when $X$ is small (cf. Figures 1 and 2) . Formula (9.3) offers a possible explanation for this. Suppose that $t=\gamma$, the ordinate of a zero of $\zeta(s)$ with multiplicity $m$. Then $N(\gamma)$ is an integer, so by (9.3)

$$
\frac{1}{2 \pi} F_{X}^{*}(\gamma) \equiv-\frac{1}{\pi} \operatorname{Im} \sum_{\gamma^{\prime}} F_{2}\left(i\left(\gamma-\gamma^{\prime}\right) \log X\right)+O\left(\gamma^{-2}\right) \quad(\bmod 1)
$$

Now, a more precise version of (3.11) is that if $y$ is real,

$$
\operatorname{Im} F_{2}(i y)=\arg i y+\sum_{k=0}^{\infty} a_{k} y^{2 k+1}
$$

where the $a_{k}$ are real and the argument is $\pi / 2$ when $y=0$ (the limit as $y \rightarrow$ $0^{+}$). This is an odd function (for $y \neq 0$ ). Furthermore, for larger $y$ we have $\operatorname{Im} F_{2}(i y)=\left(\sin y / y^{2}\right)(1+O(1 /|y|))$ by (3.21). Thus, the $m$ terms in the sum with $\gamma^{\prime}=\gamma$ contribute $m(\pi / 2)$ and the terms with $\left|\gamma-\gamma^{\prime}\right| \log X$ large are decreasing and oscillating. They should therefore exhibit a lot of cancellation. There might also be a great deal of cancellation among the small and intermediate range terms for the following reason. Although it has never been proved, we expect the numbers $\gamma^{\prime} \log X$ to be uniformly distributed $(\bmod 1)$ when $T \ll X \ll T^{A}$ with $A$ arbitrarily large and fixed. This and the fact that $\operatorname{Im} F_{2}(i y)$ is odd suggest that all the terms with $\gamma^{\prime} \neq \gamma$ cancel and that $(1 / 2 \pi) F_{X}^{*}(\gamma)$ will be close to $m / 2(\bmod 1)$. Thus, if $m$ is odd (it is believed that $m$ is always 1 ), it would not be surprising to find a zero of $\zeta_{X}^{*}\left(\frac{1}{2}+i t\right)$ nearby.

While writing this paper, the author learned from a lecture by J. P. Keating that he and E. B. Bogomolny had worked with a function similar to $\zeta_{t / 2 \pi}$ restricted to the critical line as a heuristic tool to calculate the pair correlation function of the zeros of $\zeta(s)$ (see, for example, Bogomolny and Keating [4] and Bogomolny [1]). In fact, Professor Keating [18] had first considered such a function in the early 1990s and observed that its zeros are quite close to those of the zeta-function. He and his graduate student, Steve Banham, also heuristically investigated how close the zeros of $\zeta_{X}\left(\frac{1}{2}+i t\right)$ and $\zeta\left(\frac{1}{2}+i t\right)$ are as a function of $X$.

\section{Why ZEROS OF $\zeta_{X}(s)$ ARE SIMPLE AND REPEL}

Theorem 8.1 shows that if the Riemann Hypothesis holds, then the zeros of $\zeta_{X}\left(\frac{1}{2}+i t\right)$ with $t \geq C_{0}$ are simple provided that $X \leq \exp \left(C_{3} \log t / \Phi(t)\right)$ for some constant $C_{3}>0$. Futhermore Theorem 8.2 shows unconditionally that even for $X$ as large as $\exp \left(o\left(\log ^{1-\epsilon} t\right)\right), 100 \%$ of the zeros are simple. The structure of $\zeta_{X}\left(\frac{1}{2}+i t\right)$ suggests why. The zeros of $\zeta_{X}\left(\frac{1}{2}+i t\right)$ for $t \geq C_{0}$ arise as the solutions of the congruence $F_{X}(t) \equiv \pi(\bmod 2 \pi)$. That is, they are the abscissas of the points where the curve $y=F_{X}(t)$ crosses the equally spaced horizontal lines $y=$ $(2 k+1) \pi$. If $t$ were the abscissa of a multiple zero of $\zeta_{X}\left(\frac{1}{2}+i t\right)$, it would also have to be a solution of the equation $F_{X}^{\prime}(t)=0$. We saw that this cannot happen for $X \leq \exp \left(C_{3} \log t / \Phi(t)\right)$ (because then $F_{X}^{\prime}(t)>0$ ) and that it cannot happen often if $\log X=o(\log \tau)$. However, for larger $X$, say $X \leq t^{2}$, one might also expect it to happen rarely on the grounds that the "events"

$$
F_{X}(t)=t \log \frac{t}{2 \pi}-t-\frac{1}{4} \pi-2 \sum_{n \leq X^{2}} \frac{\Lambda_{X}(n) \sin (t \log n)}{n^{\frac{1}{2}} \log n}+O\left(\frac{1}{\tau}\right) \equiv \pi(\bmod 2 \pi)
$$



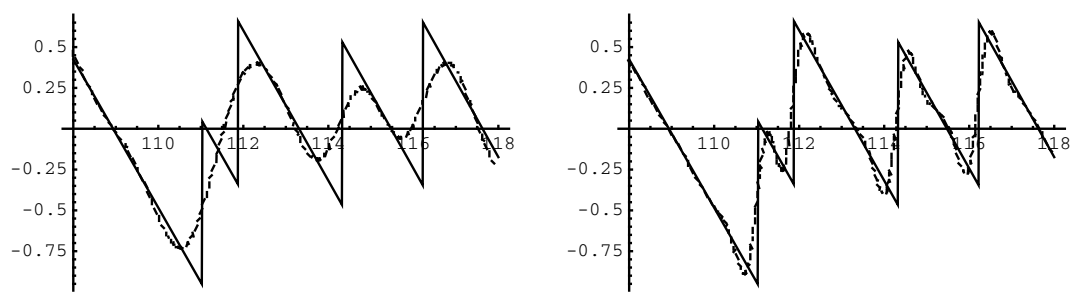

Figure 3. Graphs of $S(t)$ (solid) and $(-1 / \pi) f_{X}(t)$ (dotted) near $t=114$ for $X=10$ and $X=300$, respectively.

and

$$
F_{X}^{\prime}(t)=\log \frac{t}{2 \pi}-2 \sum_{n \leq X^{2}} \frac{\Lambda_{X}(n) \cos (t \log n)}{n^{\frac{1}{2}}}+O\left(\frac{1}{\tau}\right)=0
$$

should be independent.

What about repulsion? By (7.12) and Theorem 7.4, $F_{X}^{\prime}(t) \ll \Phi(t) \log X+\log t$ when $2 \leq X \leq t^{2}$. As in Section 7, we divide the zeros into two kinds. The first kind comes about by $y=F_{X}(t)$ increasing or decreasing from $y=(2 k+1) \pi$ to the next larger or smaller odd multiple of $\pi$ without first re-crossing $y=(2 k+1) \pi$. All other zeros are zeros of the second kind. Suppose that $\gamma_{X}$ and $\gamma_{X}^{\prime}$ are ordinates of consecutive zeros of $\zeta_{X}\left(\frac{1}{2}+i t\right)$ and that $\frac{1}{2}+i \gamma_{X}$ is a zero of the first kind. Then $F_{X}\left(\gamma_{X}^{\prime}\right)-F_{X}\left(\gamma_{X}\right)= \pm 2 \pi$ and we have

$$
\begin{aligned}
2 \pi & =\left|F_{X}\left(\gamma_{X}^{\prime}\right)-F_{X}\left(\gamma_{X}\right)\right|=\left|\int_{\gamma_{X}}^{\gamma_{X}^{\prime}} F_{X}^{\prime}(u) d u\right| \\
& \ll\left(\gamma_{X}^{\prime}-\gamma_{X}\right)\left(\log \gamma_{X}+\Phi\left(\gamma_{X}\right) \log X\right) .
\end{aligned}
$$

Thus,

$$
\gamma_{X}^{\prime}-\gamma_{X} \gg \frac{1}{\log \gamma_{X}+\Phi\left(\gamma_{X}\right) \log X} .
$$

Recall that $\left(\log \gamma_{X} / \log \log \gamma_{X}\right)^{1 / 2} \ll \Phi\left(\gamma_{X}\right) \ll \log \gamma_{X}$. Thus, if $X \leq \gamma_{X}^{2}$, then

$$
\gamma_{X}^{\prime}-\gamma_{X} \gg \frac{1}{\log ^{a} \gamma_{X}}
$$

for some $a \in\left[\frac{3}{2}, 2\right]$.

Note that if $X \leq \exp \left(C_{3} \log t / \Phi(t)\right)$ with $C_{3}$ as in Theorem 8.1, then $F_{X}^{\prime}(t)>0$ and all zeros are of the first kind. Furthermore, by the proof of Theorem[7.6] there are $(1+o(1))(t / 2 \pi) \log (t / 2 \pi)$ zeros of the first kind when $\log X=o(\log \tau)$.

If $\frac{1}{2}+i \gamma_{X}$ is a zero of the second kind, then $F_{X}\left(\gamma_{X}^{\prime}\right)-F_{X}\left(\gamma_{X}\right)=0$ and the argument above does not work. It may be, however, that most zeros are of the first kind.

To see why, first observe that $S(t)$ is a saw-tooth function because $N(t)$ is a stepfunction consisting of the increasing function $(t / 2 \pi) \log (t / 2 \pi)-(t / 2 \pi)+7 / 8+O(1 / \tau)$ plus $S(t)$. Now between consecutive ordinates $\gamma, \gamma^{\prime}$ of zeros of $\zeta\left(\frac{1}{2}+i t\right), S(t)$ decreases essentially linearly with slope $-(1 / 2 \pi) \log (\gamma / 2 \pi)$. It then jumps at $\gamma^{\prime}$ by an amount equal to the multiplicity of the zero $\frac{1}{2}+i \gamma^{\prime}$. The heuristic argument at the end of the last section suggesting that $\sum_{\gamma} F_{2}(i(t-\gamma) \log X)$ is usually small 

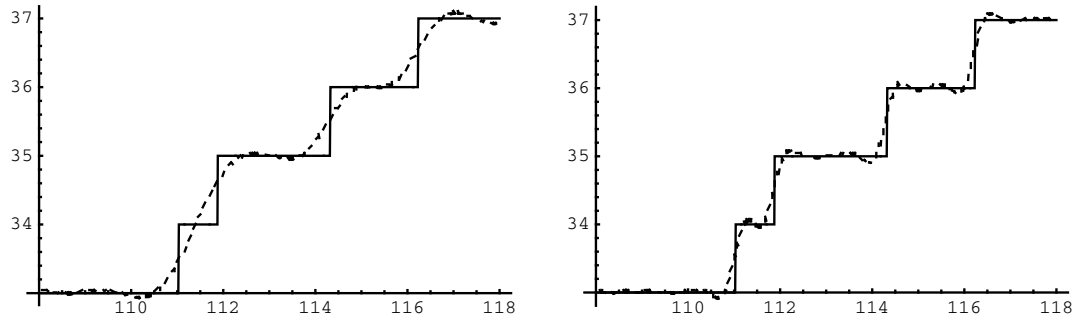

Figure 4. Graphs of $N(t)$ and $(1 / 2 \pi) F_{X}(t)+1$ near $t=114$ for $X=10$ and $X=300$

away from ordinates of zeta zeros, when applied to (9.2) with $X \leq t^{2}$, implies that between the ordinates of zeros

$$
S(t) \approx-\frac{1}{\pi} f_{X}(t)=-\frac{1}{\pi}\left(\sum_{n \leq X^{2}} \frac{\Lambda_{X}(n) \sin (t \log n)}{\sqrt{n} \log n}\right) .
$$

It is not clear here how large $X$ should be relative to $t$. However, graphs of $f_{X}(t)$ indicate that they are close to the graph of $S(t)$ when $X$ is moderately large, there are small oscillations along the downward slopes of $S(t)$, and then a flatter, close to vertical rise near the jumps of $S(t)$ (Figure 3 ). For

$$
\frac{F_{X}(t)}{2 \pi} \approx \frac{t}{2 \pi} \log \frac{t}{2 \pi}-\frac{t}{2 \pi}-\frac{1}{8}-\frac{1}{\pi} f_{X}(t),
$$

which approximates $N(t)-1$, this means that the oscillations tend to be along the flat part of the "steps" and not at the rise (Figure 4 4$)$. However, zeros of $\zeta_{X}\left(\frac{1}{2}+i t\right)$ correspond to solutions of $F_{X}(t) / 2 \pi \equiv \frac{1}{2}(\bmod 1)$, and these will be the abscissas of points that are about halfway up the rise of $F_{X}(t)$. This suggests that zeros of the second kind are unlikely.

Our arguments have assumed that $X \leq t^{2}$, but we should probably take $X$ even larger to model the zeta-function. In that case we would need to repeat the arguments with $F_{X}^{*}(t)$. This introduces a term $-\operatorname{Im} F_{2}\left(\left(-\frac{1}{2}+i t\right) \log X\right)$, which can be as large as $X / t^{2} \log ^{2} X$. Applied to the argument for gaps between zeros of the first kind with ordinates around $t$, and assuming $X=t^{b}$ with $b>2$, this leads to

$$
\gamma_{X}^{\prime}-\gamma_{X} \gg \frac{1}{\Phi_{X}\left(\gamma_{X}\right) \log \gamma_{X}+\gamma_{X}^{b-3} / \log ^{2} \gamma_{X}}
$$

in place of (10.1). This is again $\gg 1 / \log ^{a} \gamma_{X}$ for some $a \in\left[\frac{3}{2}, 2\right]$ when $2<b \leq 3$, but it gives

$$
\gamma_{X}^{\prime}-\gamma_{X} \gg 1 / \gamma_{X}^{b-3}
$$

when $b>3$. This may be compared with the repulsion between zeros of the zetafunction predicted by extrapolating from Montgomery's pair correlation conjecture, namely

$$
\gamma_{X}^{\prime}-\gamma_{X} \gg 1 / \gamma_{X}^{\frac{1}{3}+\epsilon}
$$

It would have been nice if the exponent $\frac{1}{3}$ had been forced on us by the above argument. 


\section{Other L-FunCtions AND SUMS OF L-FUnCtions}

The ideas above have obvious extensions to the more general setting of the Selberg Class of L-functions [23] and similar classes of functions with Euler products and functional equations, such as that defined by Iwaniec and Kowalski [17]. Here we briefly indicate how this looks for Dirichlet L-functions. We then consider the analogue of the problem of the distribution of zeros of linear combinations of L-functions. This approach provides a new heuristic explanation for why such combinations should have almost all their zeros on the critical line.

Let $L(s, \chi)$ denote the Dirichlet L-function with character $\chi$ modulo $q$ and functional equation

$$
L(s, \chi)=\frac{\tau(\chi)}{i^{\mathfrak{a}} \sqrt{q}} \Psi(s) L(1-s, \bar{\chi}),
$$

where

$$
\Psi(s)=\left(\frac{\pi}{q}\right)^{s-\frac{1}{2}} \frac{\Gamma\left(\frac{1+\mathfrak{a}-s}{2}\right)}{\Gamma\left(\frac{\mathfrak{a}+s}{2}\right)},
$$

$\tau(\chi)$ is Gauss' sum, and $\mathfrak{a}=0$ or 1 according to whether $\chi(-1)=1$ or -1 . When $q=1$, this is the functional equation for $\zeta(s)$ because $\tau(\chi)=1$ and $\mathfrak{a}=0$. Since the factor preceding $\Psi(s)$ has modulus 1 , we may rewrite (11.1) as

$$
e^{i \alpha} L(s, \chi)=\Psi(s) e^{-i \alpha} L(1-s, \bar{\chi}) \text {, }
$$

where $\alpha=\alpha(\chi) \in \mathbb{R}$.

We now define the functions

$$
L_{X}(s, \chi)=P_{X}(s, \chi)+e^{-2 i \alpha} \Psi(s) P_{X}(\bar{s}, \bar{\chi}),
$$

where

$$
P_{X}(s, \chi)=\exp \left(\sum_{n \leq X^{2}} \frac{\Lambda_{X}(n) \chi(n)}{n^{s} \log n}\right) .
$$

Observe that $P_{X}(\bar{s}, \bar{\chi})=\overline{P_{X}(s, \chi)}$. Clearly theorems corresponding to those we have proved for $\zeta_{X}(s)$ hold for $L_{X}(s, \chi)$. In particular, one can show that all zeros in $\frac{1}{2}<\sigma \leq 1$ have an imaginary part $\leq C_{0}$. Further, if the Riemann Hypothesis holds for $L(s, \chi)$, then $N_{X}(t, \chi)$, the number of zeros of $L_{X}\left(\frac{1}{2}+i u, \chi\right)$ with $0 \leq u \leq t$, satisfies

$$
N_{X}(t, \chi) \geq \frac{t}{2 \pi} \log \frac{q t}{2 \pi}-\frac{t}{2 \pi}+O(\log \tau)
$$

when $2 \leq X \leq t^{2}$, and equality holds if $X$ is much smaller. (We assume $q$ is fixed.) Also, unconditionally we have $N_{X}(t, \chi)=(1+o(1))(t / 2 \pi) \log (q t / 2 \pi)$ provided $\log X=o(\log \tau)$.

A number of authors ([2], [3], [14, 23]) have studied the location of zeros of linear combinations of the type

$$
\mathcal{L}(s)=\sum_{j=1}^{J} b_{j} e^{i \alpha_{j}} L\left(s, \chi_{j}\right),
$$

for Dirichlet and other L-functions with the same functional equation, that is, having the same factor $\Psi(s)$. Here the $b_{j}$ 's are real and non-zero and the inclusion of the factors $e^{i \alpha_{j}}$ ensures that $\mathcal{L}(s)$ satisfies

$$
\mathcal{L}(s)=\Psi(s) \overline{\mathcal{L}(1-\bar{s})}
$$


Typically, $\mathcal{L}(s)$ has infinitely many zeros off the critical line but no Euler product. Bombieri and Hejhal ([2, [3] ) have shown, however, that if the Riemann Hypothesis holds for each of the L-functions and their zeros satisfy a plausible spacing hypothesis, then $100 \%$ of their zeros (in the sense of density) are on the line. In the case of Dirichlet and certain other L-functions, Selberg (unpublished) has shown unconditionally that such combinations have a positive proportion of their zeros on the critical line.

The idea leading to these results was first suggested by H. L. Montgomery and is roughly as follows. Consider the case of two distinct Dirichlet L-functions $L\left(s, \chi_{1}\right)$ and $L\left(s, \chi_{2}\right)$ to the same modulus $q$ and having the same functional equation, and let $f_{1}(t)=\log \left|L\left(s, \chi_{1}\right)\right| /\left(\frac{1}{\pi} \log \log t\right)^{\frac{1}{2}}$ and $f_{2}(t)=\log \left|L\left(s, \chi_{2}\right)\right| /\left(\frac{1}{\pi} \log \log t\right)^{\frac{1}{2}}$. It is known that $f_{1}(t)$ and $f_{2}(t)$ behave like independent normally distributed random variables with mean 0 and standard deviation 1 . Thus, asymptotically half of the time on $[T, 2 T]$ we should expect the first function to be much larger than the second, and the other half of the time much smaller. It can also be shown that in any interval $\mathcal{I}$ of length $\exp (\sqrt{\log \log T}) / \log T$, one function dominates the other except possibly on a subset of measure $o(\mathcal{I})$. Suppose then that $f_{1}(t)$ dominates in $\mathcal{I}$. Then $\left|b_{1} e^{i \alpha_{1}} L\left(s, \chi_{1}\right)+b_{2} e^{i \alpha_{2}} L\left(s, \chi_{2}\right)\right|$ is essentially the size of $\left|b_{1} L\left(s, \chi_{1}\right)\right|$ and, if the zeros of $L\left(s, \chi_{1}\right)$ are well-spaced, the zeros of $b_{1} e^{i \alpha_{1}} L\left(s, \chi_{1}\right)+b_{2} e^{i \alpha_{2}} L\left(s, \chi_{2}\right)$ will be perturbations of the zeros of $L\left(s, \chi_{1}\right)$. Thus, if all or almost all of the zeros of each L-function is on the critical line, almost all the zeros of the sum should be as well.

We now ask what happens if we replace each $L$-function in the linear combination by the corresponding function $L_{X}\left(s, \chi_{j}\right)$. Set

$$
\mathcal{L}_{X}(s)=\sum_{j=1}^{J} b_{j} e^{i \alpha_{j}} L_{X}\left(s, \chi_{j}\right),
$$

where the $b_{j}$ are in $\mathbb{R}-\{0\}$, and let $\mathcal{N}_{X}(t)$ denote the number of zeros of $\mathcal{L}_{X}(s)$ on $\sigma=\frac{1}{2}$ up to height $t$. Using the definition of $\mathcal{L}_{X}(s)$ we write this as

$$
\begin{aligned}
\mathcal{L}_{X}(s) & =\sum_{j=1}^{J} b_{j} e^{i \alpha_{j}} P_{X}\left(s, \chi_{j}\right)+\Psi(s) \overline{\sum_{j=1}^{J} b_{j} e^{i \alpha_{j}} P_{X}\left(s, \chi_{j}\right)} \\
& =\mathcal{P}_{X}(s)+\Psi(s) \overline{\mathcal{P}_{X}(s)} .
\end{aligned}
$$

Clearly $\mathcal{L}_{X}(s)$ has zeros on $\sigma=\frac{1}{2}$ if either

(1) $\mathcal{P}_{X}\left(\frac{1}{2}+i t\right)=0$

or

(2) $\mathcal{F}_{X}(t)=\arg \Psi\left(\frac{1}{2}+i t\right)-2 \arg \mathcal{P}_{X}\left(\frac{1}{2}+i t\right) \equiv \pi(\bmod 2 \pi)$.

For the moment let us pass over the first case and count the number of points at which the second case happens but the first does not. By (11.2)

$$
\arg \Psi(s)=-t \log \frac{t q}{2 \pi}+t-c_{0}+O\left(\frac{1}{\tau}\right)
$$

with $c_{0}$ a real number. Thus, (2) happens at least

$$
\frac{t}{2 \pi} \log \frac{t q}{2 \pi}-\frac{t}{2 \pi}-2 \arg \mathcal{P}_{X}\left(\frac{1}{2}+i t\right)+O(1)
$$

times on $[0, t]$. Here we define $\arg \mathcal{P}_{X}\left(\frac{1}{2}+i t\right)$ by continuous variation from some point $\sigma_{0}>1$ on the real axis up to $\sigma_{0}+i t$ and then over to $\frac{1}{2}+i t$, with our usual 
convention if $\mathcal{P}_{X}$ vanishes at $\frac{1}{2}+i t$. To bound $\arg \mathcal{P}_{X}\left(\frac{1}{2}+i t\right)$, the point $\sigma_{0}$ requires some consideration. For each $j$ write

$$
P_{X}\left(s, \chi_{j}\right)=\exp \left(\sum_{n \leq X^{2}} \frac{\Lambda_{X}(n) \chi_{j}(n)}{n^{s} \log n}\right)=\sum_{n=1}^{\infty} \frac{a(n) \chi_{j}(n)}{n^{s}} .
$$

Since $0 \leq \Lambda_{X}(n) \leq \Lambda(n)$, we see that for $\sigma>1$,

$$
\begin{aligned}
\sum_{n=1}^{\infty} \frac{a(n)}{n^{\sigma}} & =\exp \left(\sum_{n \leq X^{2}} \frac{\Lambda_{X}(n)}{n^{\sigma} \log n}\right) \\
& \leq \exp \left(\sum_{n=2}^{\infty} \frac{\Lambda(n)}{n^{\sigma} \log n}\right)=\zeta(\sigma) .
\end{aligned}
$$

In particular, $0 \leq a(n) \leq 1$ and $a(1)=1$.

Next write

$$
\mathcal{P}_{X}(s)=\sum_{j=1}^{J} b_{j} e^{i \alpha_{j}} P_{X}\left(s, \chi_{j}\right)=\sum_{n=1}^{\infty} \frac{a(n)}{n^{s}}\left(\sum_{j=1}^{J} b_{j} e^{i \alpha_{j}} \chi_{j}(n)\right)=\sum_{n=1}^{\infty} \frac{a(n) B(n)}{n^{s}},
$$

say, and assume from now on that $B(1) \neq 0$. (If $B(1)=0$, the following argument would have to be modified slightly, and the number of zeros would change by $O(t)$.) Setting $B=\sum_{j=1}^{J}\left|b_{j}\right|$, we have $|B(n)| \leq B$ for every $n$, and there exists a positive constant $c_{1}$ and a real number $\omega$ such that $B(1)=c_{1} e^{i \omega} B$. It follows that for $\sigma>1$,

$$
\operatorname{Re}\left(e^{-i \omega} \mathcal{P}_{X}(s)\right) \geq c_{1} B-B \sum_{n=2}^{\infty} \frac{1}{n^{\sigma}} \geq B\left(c_{1}-\int_{1}^{\infty} x^{-\sigma}\right)=B\left(c_{1}-\frac{1}{\sigma-1}\right) .
$$

This is positive if $\sigma>1+1 / c_{1}$. Thus, if $\sigma_{0}$ meets this condition,

$$
\operatorname{Re}\left(e^{-i \omega} \mathcal{P}_{X}\left(\sigma_{0}+i t\right)\right)>0
$$

for all $t$, and $\arg \mathcal{P}_{X}\left(\sigma_{0}+i t\right)$ varies by at most $\pi$ on $\left[\sigma_{0}, \sigma_{0}+i t\right]$. It follows that $\left|\arg \mathcal{P}_{X}\left(\frac{1}{2}+i t\right)\right|$ is less than or equal to the change in argument of $\mathcal{P}_{X}(s)$ on the segment $\left[\frac{1}{2}+i t, \sigma_{0}+i t\right]$ plus $\pi$. By a well-known lemma in Section 9.4 of Titchmarsh [25], if $\left|\mathcal{P}_{X}\left(\sigma^{\prime}+i t^{\prime}\right)\right| \leq M(\sigma, t)$ for $\frac{1}{2} \leq \sigma \leq \sigma^{\prime}, 1 \leq t^{\prime} \leq t$, then this change in $\operatorname{argument}$ is $\ll_{\epsilon} \log \left(M\left(\frac{1}{2}-\epsilon, \tau\right) /\left|\operatorname{Re} e^{-i \omega} \mathcal{P}_{X}\left(\sigma_{0}\right)\right|\right)+1$ for any $\epsilon>0$. Now

$$
\left|\mathcal{P}_{X}(s)\right| \leq B \sum_{j=1}^{J}\left|P_{X}\left(s, \chi_{j}\right)\right|
$$

and

$$
P_{X}\left(s, \chi_{j}\right) \ll \exp \left(\sum_{n \leq X^{2}} \frac{\Lambda_{X}(n)}{n^{\sigma} \log n}\right) \ll \exp \left(\frac{X^{2(1-\sigma)}}{\log X}\right) .
$$

Thus,

$$
\arg \mathcal{P}_{X}\left(\frac{1}{2}+i t\right) \ll_{\epsilon} \frac{X^{1+2 \epsilon}}{\log X} .
$$

This is a very crude bound, but it suffices here. By (11), we now have

$$
\mathcal{N}_{X}(t) \geq \frac{t}{2 \pi} \log \frac{t q}{2 \pi}-\frac{t}{2 \pi}+O_{\epsilon}\left(X^{1+2 \epsilon}\right),
$$

and the leading term is larger than the $O$-term if $X<t^{1-2 \epsilon}$. To leading order this is also the lower bound for the number of zeros of each $L_{X}(s, \chi)$. With more work 
we could show unconditionally that when $\log X / \log \tau=o(1)$, the number of zeros arising from case (2) is in fact $=(1+o(1))(t / 2 \pi) \log (t / 2 \pi)$.

An analysis of the contribution of zeros from case (1) is rather elaborate, and we will not attempt it here. One expects relatively few zeros to arise in this way, though, because it is unlikely that the curve $z=\mathcal{P}_{X}\left(\frac{1}{2}+i t\right)$ will pass through the origin. As with our previous results, the difficulty we have is not in proving that there are lots of zeros on the line, but that there are not too many, and, just as before, we have only limited success with this.

The main point is that one can see immediately from the structure of $L_{X}(s, \chi)$ why one might expect $100 \%$ of the zeros of linear combinations of such functions to lie on the critical line. It therefore suggests a reason this should be true for linear combinations of actual L-functions, and this reason is different from the usual one.

\section{Appendix}

Theorem. A necessary and sufficient condition for the truth of the Lindelö Hypothesis is that for $\frac{1}{2} \leq \sigma \leq 2,|s-1|>\frac{1}{10}$, and $2 \leq X \leq \tau^{2}$,

$$
\zeta(s)=\sum_{n \leq X} \frac{1}{n^{s}}+O\left(X^{\frac{1}{2}-\sigma} \tau^{\epsilon}\right) .
$$

Moreover, if the Riemann Hypothesis is true, then there exists a positive constant $C_{1}$ such that for $X$ and $s$ as above,

$$
\zeta(s)=\sum_{n \leq X} \frac{1}{n^{s}}+O\left(X^{\frac{1}{2}-\sigma} e^{C_{1} \Phi(t)}\right) .
$$

Here $\Phi(t)$ is an admissible function in the sense of Section 3. In particular, we have

$$
\zeta(s) \ll e^{C_{1} \Phi(t)}
$$

for $\frac{1}{2} \leq \sigma \leq 2$ and $|s-1|>\frac{1}{10}$.

Proof. The proof of a statement similar to the first assertion may be found in Titchmarsh 25] (Theorem 13.3). Moreover, the more difficult implication (Lindelöf implies (12.1)) is proved by an easy modification of the proof of the second assertion, which we turn to now.

We apply Perron's formula (Lemma 3.19 of Titchmarsh [25) to $\zeta(s)$ and obtain

$$
\sum_{n \leq X} \frac{1}{n^{s}}=\frac{1}{2 \pi i} \int_{c-i U}^{c+i U} \zeta(s+w) \frac{X^{w}}{w} \mathrm{~d} w+O\left(\frac{X^{\frac{1}{2}} \log 2 X}{U}\right)+O\left(X^{-\sigma}\right),
$$

where $X \geq 2$ and $c=\frac{1}{2}+\frac{1}{\log X}$. Letting $b=\frac{1}{2}-\sigma-\frac{1}{\log \tau}$ and $\mathcal{R}$ the positively oriented rectangle with vertices $b \pm i U$ and $c \pm i U$, we find that

$$
\frac{1}{2 \pi i} \int_{\mathcal{R}} \zeta(s+w) \frac{X^{w}}{w} \mathrm{~d} w=\zeta(s)+\frac{X^{1-s}}{1-s} .
$$

On the Riemann Hypothesis,

$$
\left(\int_{b-i U}^{c-i U}+\int_{c+i U}^{b+i U}\right) \zeta(s+w) \frac{X^{w}}{w} \mathrm{~d} w \ll X^{\frac{1}{2}} e^{\Phi(U+\tau)} U^{-1} .
$$


Here we have used the functional equation and the estimates $\left|\zeta\left(\frac{1}{2}+\frac{1}{\log \tau}+i(t+v)\right)\right| \ll$ $e^{\Phi(U+\tau)}$ and $X^{c} \ll X^{\frac{1}{2}}$. Also by the Riemann Hypothesis,

$$
\begin{aligned}
\int_{b-i U}^{b+i U} \zeta(s+w) \frac{X^{w}}{w} \mathrm{~d} w & \ll X^{\frac{1}{2}-\sigma} e^{\Phi(U+\tau)} \int_{0}^{U}\left(b^{2}+(v)^{2}\right)^{-\frac{1}{2}} d v \\
& \ll X^{\frac{1}{2}-\sigma} e^{\Phi(U+\tau)} \log (U / b) \\
& \ll X^{\frac{1}{2}-\sigma} e^{\Phi(U+\tau)} \log U .
\end{aligned}
$$

Combining (12.4) - (12.7), we obtain

$$
\zeta(s)=\sum_{n \leq X} \frac{1}{n^{s}}+\frac{X^{1-s}}{s-1}+O\left(\frac{X^{\frac{1}{2}} e^{\Phi(U+\tau)}}{U}\right)+O\left(X^{\frac{1}{2}-\sigma} e^{\Phi(U+\tau)} \log \tau\right) .
$$

Since $X \leq \tau^{2}$ the second term on the right is $\ll X^{\frac{1}{2}-\sigma}\left(X^{\frac{1}{2}} / \tau\right) \ll X^{\frac{1}{2}-\sigma}$. The third is $\ll X^{\frac{1}{2}} e^{\Phi(U+\tau)} U^{-1} \ll X^{\frac{1}{2}-\sigma} e^{\Phi(U+\tau)}$ since $U=\tau+X^{\sigma}>X^{\sigma}$. Thus, we find that

$$
\zeta(s)=\sum_{n \leq X} \frac{1}{n^{s}}+O\left(X^{\frac{1}{2}-\sigma} e^{\Phi(U+\tau)} \log \tau\right) .
$$

Finally, by (3.13) and the fact that $\Phi$ is increasing, we have $\Phi(U+\tau) \leq \Phi\left(\tau^{4}+2 \tau\right) \leq$ $\Phi\left(2 \tau^{4}\right) \leq C_{1} \Phi(t)$. This establishes (12.2).

The bound in (12.3) follows immediately by taking $X=2$ in (12.2).

Now set

$$
S(u)=\sum_{n \leq u} \frac{1}{n^{\frac{1}{2}+i t}} .
$$

Since $\zeta\left(\frac{1}{2}+i t\right) \ll e^{\Phi(t)}$, by (12.2) we see that

$$
S(u) \ll e^{C_{1} \Phi(t)}
$$

for $1 \leq u \leq \tau^{2}$. By Stieltjes integration, if $\sigma<\frac{1}{2}$,

$$
\begin{aligned}
\sum_{n \leq X} \frac{1}{n^{\sigma+i t}} & =\int_{1^{-}}^{X} u^{\frac{1}{2}-\sigma} d S(u)=\left.u^{\frac{1}{2}-\sigma} S(u)\right|_{1^{-}} ^{X}-\left(\frac{1}{2}-\sigma\right) \int_{1}^{X} u^{-\frac{1}{2}-\sigma} S(u) d u \\
& \ll X^{\frac{1}{2}-\sigma} e^{C_{1} \Phi(t)}+\left(\frac{1}{2}-\sigma\right) e^{A_{1} \Phi(t)} \int_{1}^{X} u^{-\frac{1}{2}-\sigma} d u \\
& \ll X^{\frac{1}{2}-\sigma} e^{C_{1} \Phi(t)} .
\end{aligned}
$$

We also have from (4.1) and (12.3) that when $\sigma \geq \frac{1}{2}$,

$$
\sum_{n \leq X} \frac{1}{n^{\sigma+i t}} \ll e^{C_{1} \Phi(t)}
$$

Combining our estimates, we obtain the

Corollary. Let $1 \leq X \leq \tau^{2},|\sigma| \leq 2$, and $|s-1|>\frac{1}{10}$. If the Riemann Hypothesis is true we have

$$
\sum_{n \leq X} \frac{1}{n^{s}} \ll X^{\max \left(\frac{1}{2}-\sigma, 0\right)} e^{C_{1} \Phi(t)}
$$


Moreover, A necessary and sufficient condition for the truth of the Lindelöf Hypothesis is that

$$
\sum_{n \leq X} \frac{1}{n^{s}} \ll X^{\max \left(\frac{1}{2}-\sigma, 0\right)} \tau^{\epsilon}
$$

\section{ACKNOWLEDGEMENTS}

The author wishes to thank Enrico Bombieri, Dimitri Gioev, Jon Keating, and Peter Sarnak for very helpful conversations and communications. The author owes an especially great debt to Dimitri Gioev for many stimulating discussions and for the extensive computer calculations he performed.

\section{REFERENCES}

1. E. Bogomolny, "Spectral statistics", Doc. Math. J.-Extra vol. ICM (1998), 99-108. MR 1648144 (99h:81053)

2. E. Bombieri and D. A. Hejhal, "On the zeros of Epstein zeta functions", C. R. Acad. Sci. Paris Ser. I Math. 304 (1987), no. 9, 213-217. MR883477 (89c:11136)

3. E. Bombieri and D. A. Hejhal, "On the distribution of zeros of linear combinations of Euler products", Duke Math. J. 80 (1995), 821-862. MR.1370117(96m:11071)

4. E. B. Bogomolny and J. P. Keating, "Gutzwiller's trace formula and spectral statistics: Beyond the diagonal approximation", Phys. Rev. Lett. 77 (1996), no. 8, 1472-1475.

5. R. Balasubramaian and K. Ramachandra, "On the frequency of Titchmarsh's phenomenon for $\zeta(s)$, III", Proc. Indian Acad. of Sci. 86 A (1977), 341-351. MR0506063 (58:21968)

6. H. Davenport, Multiplicative Number Theory (2nd edition), Springer, New York, 1980. MR606931 (82m:10001)

7. D. Farmer, S. M. Gonek, C. P. Hughes, "The maximum size of L-functions", J. Reine Angew. Math. (Crelle's Journal) 609 (2007), 215-236. MR2350784 (2009b:11140)

8. D. A. Goldston and S. M. Gonek, "A note on $S(t)$ and the zeros of the Riemann zeta-function", Bull. London Math. Soc. 39 (2007), 482-486. MR2331578 (2008f:11097)

9. S. M. Gonek, "Mean values of the Riemann zeta-function and its derivatives", Invent. Math. 75 (1984), 123-141. MR728143 (85f:11063)

10. S. M. Gonek, C. P. Hughes and J. P. Keating, "A hybrid Euler-Hadamard product for the Riemann zeta function", Duke Math. J. 136, no. 3, 2007, 507-549. MR2309173(2008e:11100)

11. A. P. Guinand, "Some Fourier transforms in prime-number theory", Quart. J. Math. Oxford Ser. (2) 18 (1947), 53-64. MR.0019677 (8:447a)

12. A. P. Guinand, "A summation formula in the theory of prime numbers", Proc. London Math. Soc. (2) 50 (1948), 107-119. MR0026086(10:104g)

13. G. H. Hardy and J. E. Littlewood, "Contributions to the theory of the Riemann zeta-function and the theory of the distribution of primes", Acta Arith. 41 (1918), 119-196. MR 1555148

14. D. A. Hejhal, On a result of Selberg concerning zeros of linear combinations of L-functions, I.M.R.N., (2000), 551-577. MR.1763856 (2001m:11149)

15. C. P. Hughes, J. P. Keating and N. O'Connell, "Random matrix theory and the derivative of the Riemann zeta function", Proc. R. Soc. Lond. A 456 (2000) 2611-2627. MR 1799857 (2002e:11117)

16. A. E. Ingham, "Mean-values theorems in the theory of the Riemann zeta-function", Proc. Lond. Math. Soc. 27 (1926), 273-300.

17. H. Iwaniec and E. Kowalski, Analytic Number Theory, American Mathematical Society Colloquium Publications 53, Providence, RI, 2004. MR2061214 (2005h:11005)

18. J. P. Keating, "Quantum chaology and the Riemann zeta-function", in Quantum Chaos, eds. G. Casati, I. Guarneri, and U. Smilansky, North-Holland, Amsterdam, 1993, 145-185. MR.1246830 (95d:81029)

19. J. P. Keating and N. C. Snaith, "Random matrix theory and $\zeta(1 / 2+\mathrm{i} t)$ ", Commun. Math. Phys. 214 (2000), 57-89. MR.1794265(2002c:11107)

20. H. L. Montgomery, "Mean and large values of Dirichlet polynomials", Invent. Math. 8 (1969), 334-345. MR0268130 (42:3029) 
21. H. L. Montgomery, "The pair correlation of zeros of the zeta function", Analytic Number Theory, Proceedings of Symposia in Pure Mathematics 24 (1973), 181-193. MR 0337821 (49:2590)

22. H. L. Montgomery and R. C. Vaughan, "Hilbert's inequality", J. London Math. Soc. (2) 8 (1974), 73-82. MR0337775 (49:2544)

23. A. Selberg, "Old and new conjectures and results about a class of Dirichlet series", in Collected Papers, vol. 2, Springer Verlag, 1991, 47-63. MR1220477 (94f:11085)

24. A. Selberg, "On the remainder in the formula for $N(T)$, the number of zeros of $\zeta(s)$ in the strip $0<t<T$ ", Avhandlinger Norske Vid. Akad. Oslo, no. 1, (1944), 1-27. MR0015426 $(7: 417 \mathrm{~b})$

25. E. C. Titchmarsh, The Theory of the Riemann Zeta-Function (2nd edition, revised by D. R. Heath-Brown), Oxford Science Publications, 1986. MR882550 (88c:11049)

Department of Mathematics, University of Rochester, Rochester, New York 14627

E-mail address: gonek@math.rochester.edu 